\title{
Balance and proprioception impairment, assessment tools, and rehabilitation training in patients with total hip arthroplasty: a systematic review
}

\author{
Luciana Labanca ${ }^{1,2^{*}} \mathbb{D}$, Francesca Ciardulli ${ }^{1}$, Fabio Bonsanto ${ }^{1}$, Nadia Sommella' ${ }^{1}$, Alberto Di Martino ${ }^{2,3}$ and \\ Maria Grazia Benedetti ${ }^{1,2}$
}

\begin{abstract}
Background: Osteoarthritis and subsequent total hip arthroplasty (THA) lead to damages to hip joint mechanoceptors, which in turns lead to impairments in proprioception. One of the abilities mainly affected by an altered joint proprioception is balance. The aim of this work was to investigate the balance and proprioception impairments, current assessment tools, and rehabilitation training after THA.

Methods: A systematic literature revision was conducted on PubMed, Web of Science and Cochrane databases. Articles reporting balance and proprioception impairments, current assessment tools, or rehabilitation interventions were included. Methodological quality was assessed using the Downs and Black checklist. A total of 41 articles were included, 33 discussing balance and proprioception assessment, and 8 dealing with training. Data related to type of surgical approach, type and timing of assessment protocols, assessment instrumentation, and type, volume and duration of the rehabilitation training were extracted from each study.

Results: Thirty-one studies were of high quality, 2 of moderate quality and 8 of low-quality. Literature review showed an improvement in balance following THA in comparison with the pre-operative performance, although balance abnormalities persist up to 5 years after surgery, with THA patients showing an increased risk for falls. Balance training is effective in all the rehabilitation phases if specifically structured for balance enhancement and consistent in training volume. It remains unclear which assessments are more appropriate for the different rehabilitation phases, and if differences exist between the different surgical procedures used for THA. Only two studies assessed proprioception.

Conclusion: Balance and proprioception show impairments up to 5 years after THA, increasing the risk of falls. However, patients with THA may benefit of an adequate balance training. Further research is needed to investigate the gaps in balance and proprioception assessment and training following THA surgery.
\end{abstract}

Keywords: Total hip replacement, Hip surgery, Postural stability, Functional assessment, Risk of falls

*Correspondence: luciana.labanca88@gmail.com

2 Department of Biomedical and Neuromotor Sciences, University of Bologna, Bologna, Italy

Full list of author information is available at the end of the article

\section{Background}

Total hip arthroplasty (THA) is the most used treatment for severe hip osteoarthritis, leading patients to an immediate decrease of pain and to consistent improvements in hip joint function and quality of life [1-3]. However, osteoarthritis and major surgical original author(s) and the source, provide a link to the Creative Commons licence, and indicate if changes were made. The images or other third party material in this article are included in the article's Creative Commons licence, unless indicated otherwise in a credit line to the material. If material is not included in the article's Creative Commons licence and your intended use is not permitted by statutory regulation or exceeds the permitted use, you will need to obtain permission directly from the copyright holder. To view a copy of this licence, visit http://creativecommons.org/licenses/by/4.0/. The Creative Commons Public Domain Dedication waiver (http://creativeco mmons.org/publicdomain/zero/1.0/) applies to the data made available in this article, unless otherwise stated in a credit line to the data. 
interventions for joints replacement may compromise part of the joint structures and surrounding components, with joint mechanoceptors being the most affected structure by THA surgery. The damages to these mechanoceptors lead to impairments in proprioception, i.e., to a lack or to abnormal afferent signals informing the brain of joints' position and movement. Abnormal proprioceptive signals do not only affect sensory function, but also motor control, since sensory information is essential for movement programming [4]. For these reasons, patients undergoing joint replacement surgery show both sensitive and motor abnormalities $[2,5,6]$. One of the "abilities" mainly affected by abnormal proprioception is balance, and its impairments may compromise the quality of life [7-9], and the risk of falls [10]. In THA patients, balance deficits may be persistent after surgery [11, 12], leading to an increase in the risk of falls [13], especially in the first year after surgery [14].

The abnormalities in proprioception may also affect the biomechanics of functional movements. In fact, if afferent information is lacking, movements cannot be controlled across the whole range of motion of the joint. It is not surprising that following THA, patients show gait abnormalities up to 1 year after surgery [2]. In particular, a reduction of gait velocity and stride length have been reported, accompanied by a reduction of the time of single limb support, and a reduced range of motion in the sagittal plane [2].

Different surgical approaches for THA implant may affect hip proprioception in different ways. In fact, while the direct anterior approach (DAA) affects only hip joint capsule [15], other procedures, such as the lateral and posterior techniques, may also affect muscles and tendons [15] causing higher damages to proprioceptors. However, while the effect of different surgical approaches on hip biomechanics and clinical outcomes has been widely studied [16-19], the entity of proprioception compromise in this setting has not been extensively explored so far.

Moreover, few studies have been reported by previous systematic reviews on the benefits of balance training following THA [20], and it is not clear which kind of exercises should be adopted and how these should be differentiated in the different rehabilitation phases after THA surgery.

Having clear information on the magnitude of balance and proprioception impairments following THA would be of high clinical relevance, to ascertain which kind of balance and proprioception training interventions should be adopted, and how deficits and improvements throughout rehabilitation should be assessed.
Therefore, the current study has three main objectives. The first is to provide an updated systematic review on balance and proprioception impairments following THA surgery. The second is to investigate how balance and proprioception deficits are measured. The third objective is to investigate how balance and proprioception are trained during the rehabilitation following THA. These three points will be investigated by differentiating results according to the surgical approach used for THA implant.

\section{Methods}

\section{Population and diagnosis of interest}

This review includes all studies on balance and proprioception impairments, assessment or training after THA for degenerative arthritis.

\section{Search strategy and inclusion criteria}

A systematic review of PubMed, Web of Science, and Cochrane database was performed. The inclusion criteria were: (1) articles published between June 1, 2000, and August 31, 2021; (2) patients with THA for degenerative arthritis were recruited, (3) assessment of balance and/or proprioception, and (4) training of balance and/or proprioception. No limitations were placed over the type of surgical procedure used for THA. Non-English language publications, review articles, conference proceedings, editorials, case-studies, letters, methodological studies, animal studies, and cadaveric studies were excluded. A time frame of 20 years was chosen for two main reasons. The first, is related to the fact that methodological literature suggest to write reviews based on nearly the last $5-10$ years to be considered up-to-date $[21,22]$. Then the suggested timing was extended to 20 years to include a broader number of evidences. The second reason is related to the to the fact that studies older than 20 years may have been based on surgical procedures, as for example high invasive approaches requiring long healing time, which are no longer adopted in clinical practice to date [23]. As a consequence, the post-surgical assessment, in particular in the early post-surgery was mainly based on surgery-related consequences (e.g, pain, bleeding, ...) rather than on the assessment of functional abilities having an impact on the quality of life [23].

The terms and key words used for the research strategy were: (total hip arthroplasty OR THA OR hip replacement OR hip prosthesis) AND (balance OR propriocept* OR postural control) located within the title and/or abstract and/or keywords. The character * was used to include in the research both the terms proprioceptive, proprioception, and proprioceptors. Reference lists and citations of the included articles were manually screened to identify additional studies 
of interest. The Preferred Reporting Items for Systematic Reviews and Meta-Analyses (PRISMA) checklist was employed to guarantee review methodological quality (Additional file 1: Appendix 1). The study was registered in PROSPERO (CRD42020213412).

\section{Selection process}

Three independent reviewers (FC, FB, NS) performed the research and selection of the papers. Duplicates were removed and then the titles and abstracts of all the studies were reviewed to determine their eligibility. In case of disagreement in the appropriateness of the paper, a fourth author (LL) was consulted to determine abstracts inclusion; in that case, the full-text version of the paper was retrieved and screened to determine the eligibility of the paper. Then, the full-text of all the eligible articles was retrieved and assessed to further verify inclusion and exclusion criteria meeting. Selection process of the papers' selection is represented in Fig. 1.

\section{Risk of bias assessment}

The Quality Index Checklist by Downs and Black [24] was used to assess the risk of bias of the studies. The full version of the Downs and Black Checklist [24] was used to assess randomized and non-randomized clinical trials, while the modified version was used to assess all the other studies. The number of items of the modified version is reduced from 27 to $14(1,2,3,5,6,7,10$, $11,12,16,18,20,21,22)$ to eliminate questions that are applicable only for intervention studies. Each study was divided by quality according to the percentage of met items: low quality $(<60 \%)$, moderate quality $(61-74 \%)$, or high quality $(\geq 75 \%)$ [25]. Detailed information on the risk of bias assessment is reported in Additional file 2: Appendix 2. The reviewers assigned the judgment and the Fleiss' Kappa measure was used for assessing the reliability of agreement between the two reviewers by the use of an independent observer. This measure calculates the degree of agreement in classification compared to what would be expected by chance, and it is scored

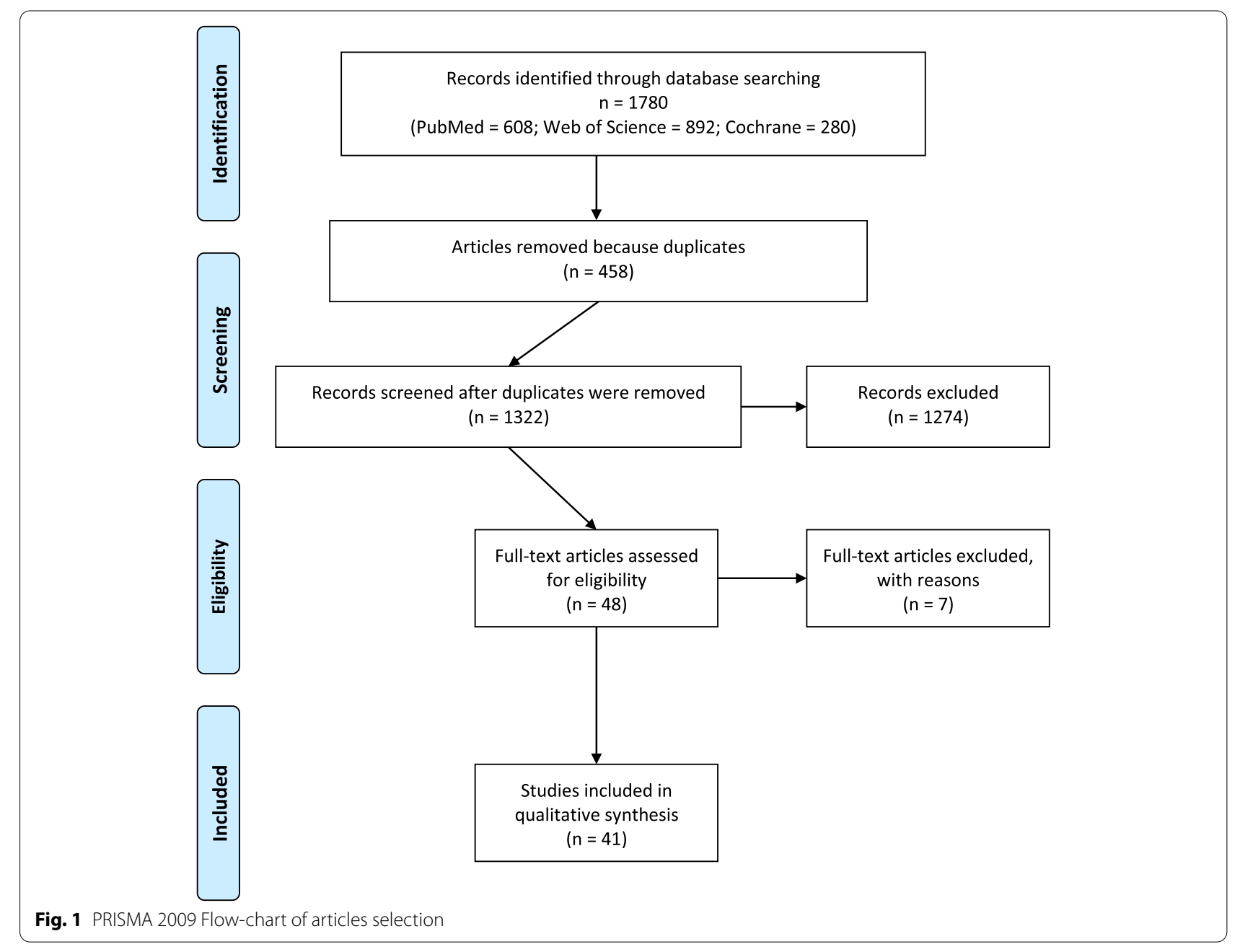


between 0 and 1 . Reliability from Kappa values was interpreted as $<0=$ Poor agreement; $0.0-0.20=$ Slight agreement; $0.21-0.40=$ Fair agreement; $0.41-0.60=$ Moderate agreement; $0.61-0.80=$ Substantial agreement and $0.81-$ $1.00=$ Almost perfect agreement, according to Landis and Koch [26].

\section{Outcomes of interest and data synthesis}

Data were extracted by three independent reviewers (FC, FB, NS), and in case of disagreement a fourth reviewer (LL) was consulted. Data extracted from studies focused on balance and proprioception assessment were: (1) authors of the study; (2) characteristics of the patients involved in the study; (3) surgical approach for THA implant; (4) the training intervention performed; (5) the protocol of assessment; (6) timepoints at which the assessments were performed; and (7) clinical results. Data extracted from the studies focused on the training of balance and proprioception were: (1) authors of the study; (2) characteristics of the patients involved in the study; (3) surgical approach for THA; (4) volume, duration and time of the training; (5) the assessments performed and timepoints in which these were performed; (6) the protocol of assessment; and (7) clinical results. If relevant data were not reported in any of the studies, the corresponding author was contacted. The data extracted from the studies and included in the qualitative synthesis are reported in Table 1 and Table 2.

\section{Deviations from the study protocol registered in PROSPERO database}

Two adjustments with respect to the original study protocol registered on PROSPERO database should be mentioned. First, the Downs and Black checklist was adopted instead of previously planned tool as it can be adapted to both intervention and non-intervention studies, and thus allowed to use a single tool for all the studies included in the present review. This choice was not made on the basis of the results of methodological assessment, rather on the willingness to provide better clarity in the reporting and the discussion of the risk of bias assessment in the text of the manuscript. The assessment with the Downs \& Black checklist and the assessment with previously planned tools was performed and the results showed comparable outcomes between the tools. Detailed information on the assessment and the comparison between the tools is reported in Additional file 3: Appendix 3. In addition, in this review there was also an attempt to analyse differences between patients undergoing different surgical approaches for THA, and this was not planned in the original study protocol. However, it was deemed appropriate to extract also these data since this information is highly useful for clinical practice and for the setting-up of the rehabilitation programs.

\section{Results \\ Search results}

According to the PRISMA flow-chart (Fig. 1), a total of 1780 articles were retrieved from the initial literature search. After removal of duplicates and of studies not meeting inclusion and exclusion criteria, 48 articles were considered eligible for a full-text review. Five articles [27, 28, 29-32] were excluded because the results from patients with THA were mixed to results of patients operated on for other orthopaedic conditions. One study was excluded because it was a reliability study [30], and another one was excluded because it did not report postsurgical data on balance assessment [33]. At the end of the selection protocol, 41 articles were included and evaluated for systematic review; of these, 33 were assessment studies [11, 13, 34-64], while the remaining 8 were rehabilitation training studies [65-72]. A summary of the data extracted from each article is reported in Table 1 and Table 2 .

\section{Studies quality and risk of bias}

The scores of the modified Quality Index for the included studies ranged between 64.2 and $100 \%$. A total of 2 studies were of moderate quality (score $60-74 \%$ ), and 31 were of high quality (score $\geq 75 \%$ ), while the remaining 8 articles were of low quality (score $<60 \%)$. The risk of bias score for each individual study is reported in Additional file 2: Appendix 2. A graphical representation of the risk of bias across all the studies reporting balance and proprioception impairments and assessments is provided in Fig. 2, while risk of bias across studies investigating rehabilitation training for balance is represented in Fig. 3. The majority of the papers included in this review (31 out of 41) were of high quality, thus showing a low risk of bias. However, regarding articles on balance and proprioception assessment, sources of high risk of bias were observed regarding the items 5, 12 and 21 of the checklists, which referred to the reporting of confounders among the patients recruited (reporting bias), the representativity of the participants recruited with respect to the whole population (external validity) and the common source population between cases and controls (selection bias), respectively. In addition, since a high percentage of study did not clearly report the required information, some concerns arise regarding items 11 and 22, i.e., the representativity of the participants which were asked to participate in the study with respect to the whole population (external validity) and the recruitment of cases and controls in the same period of time (selection bias). 


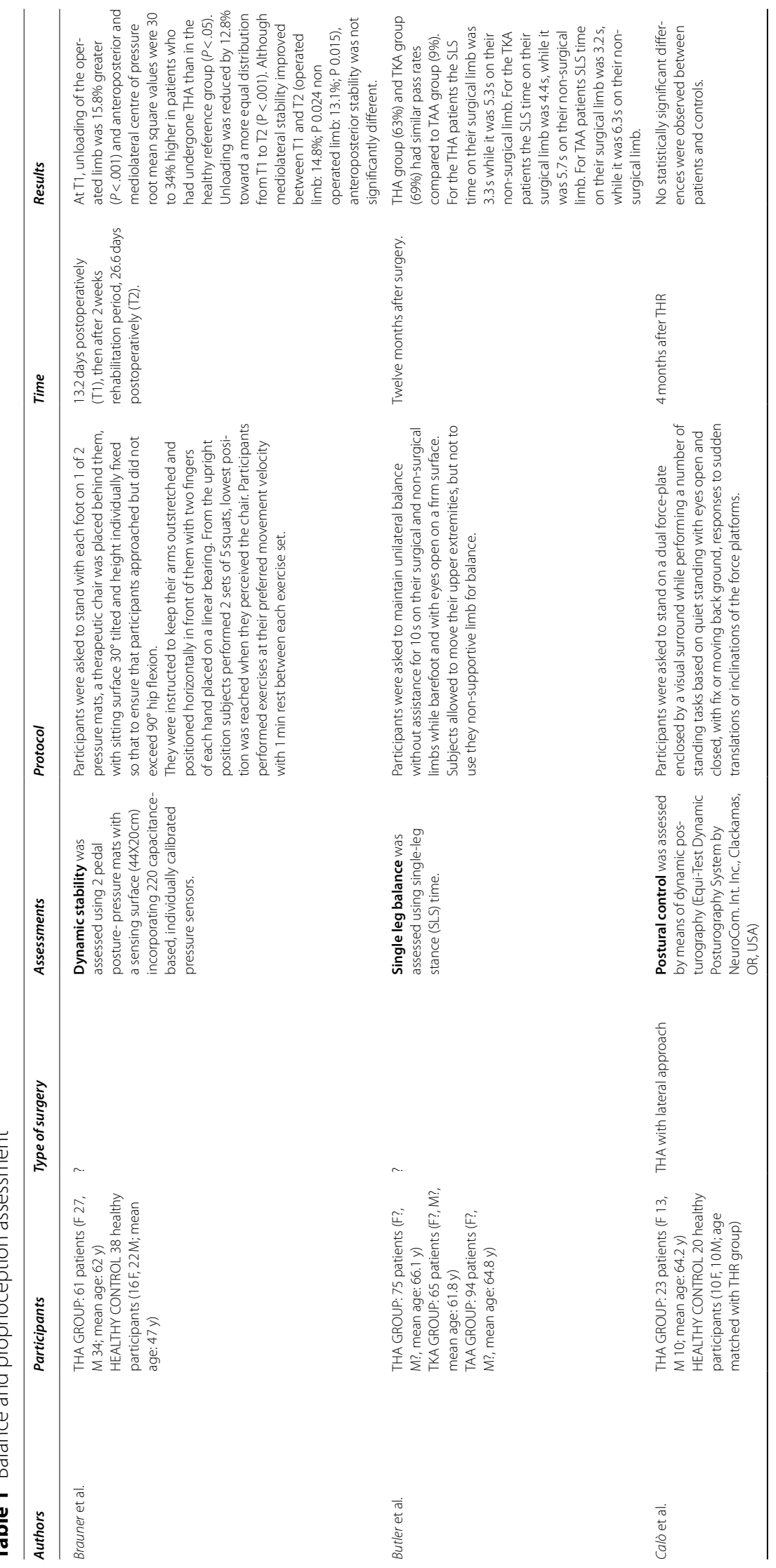




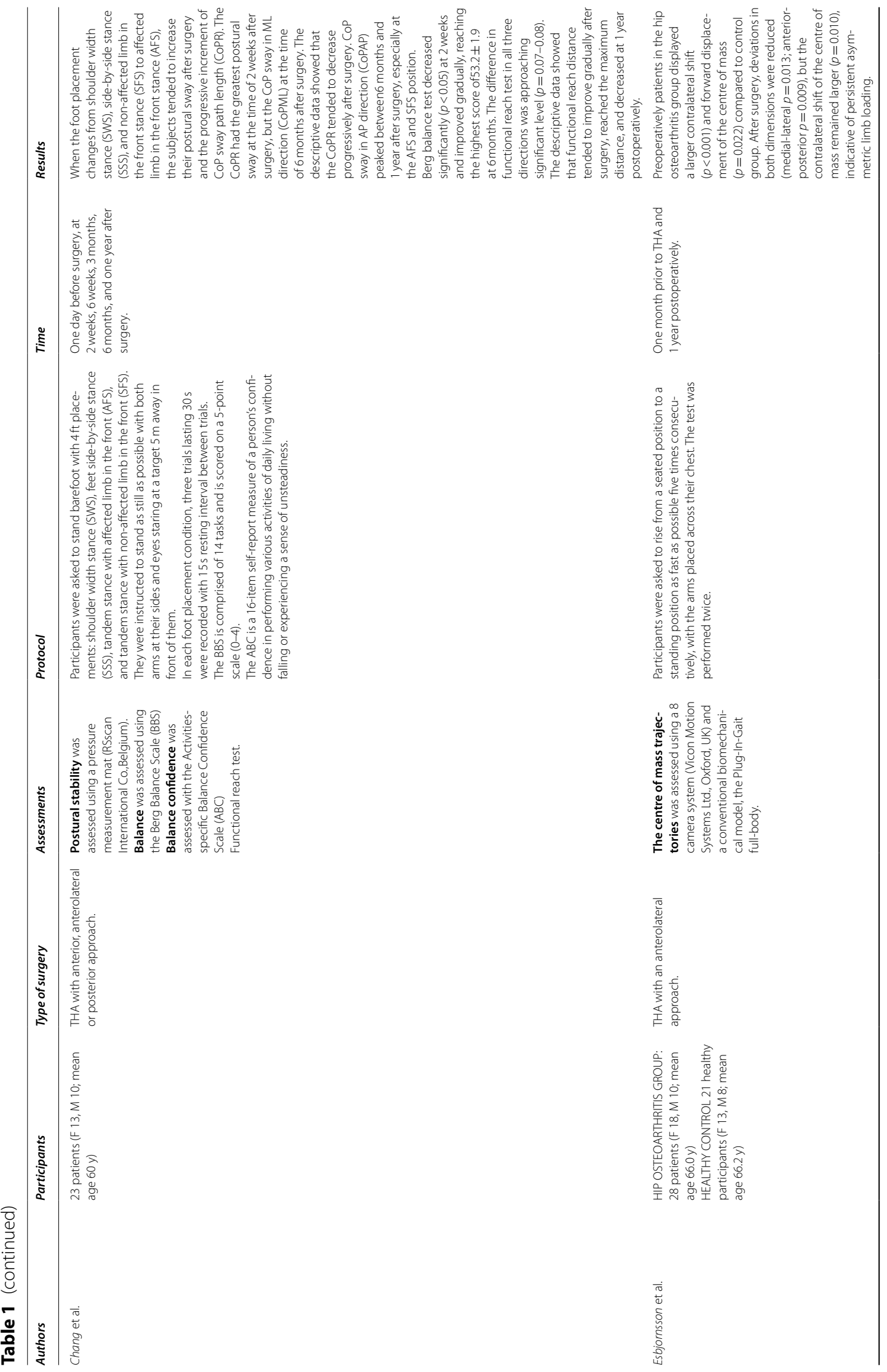




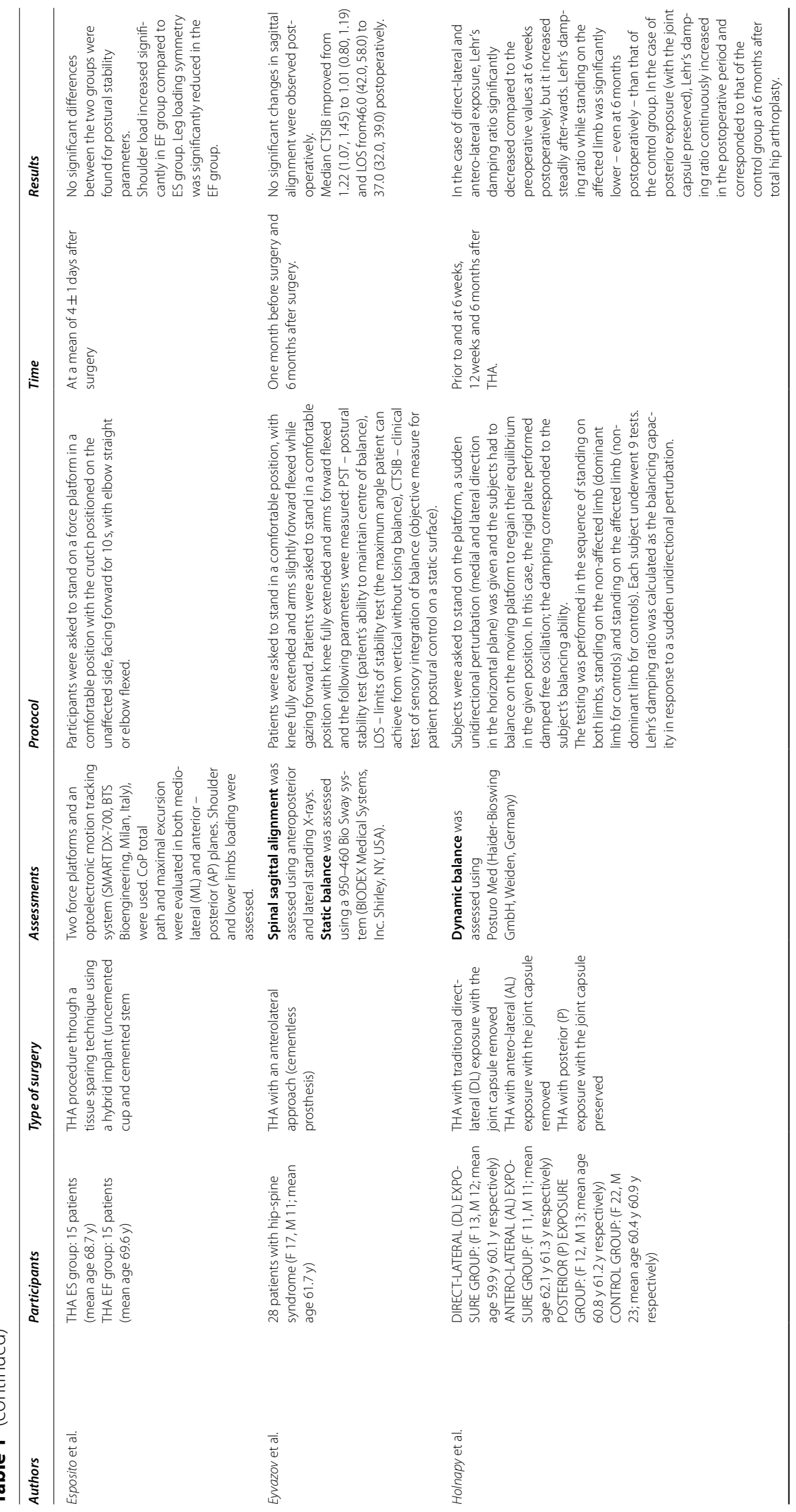




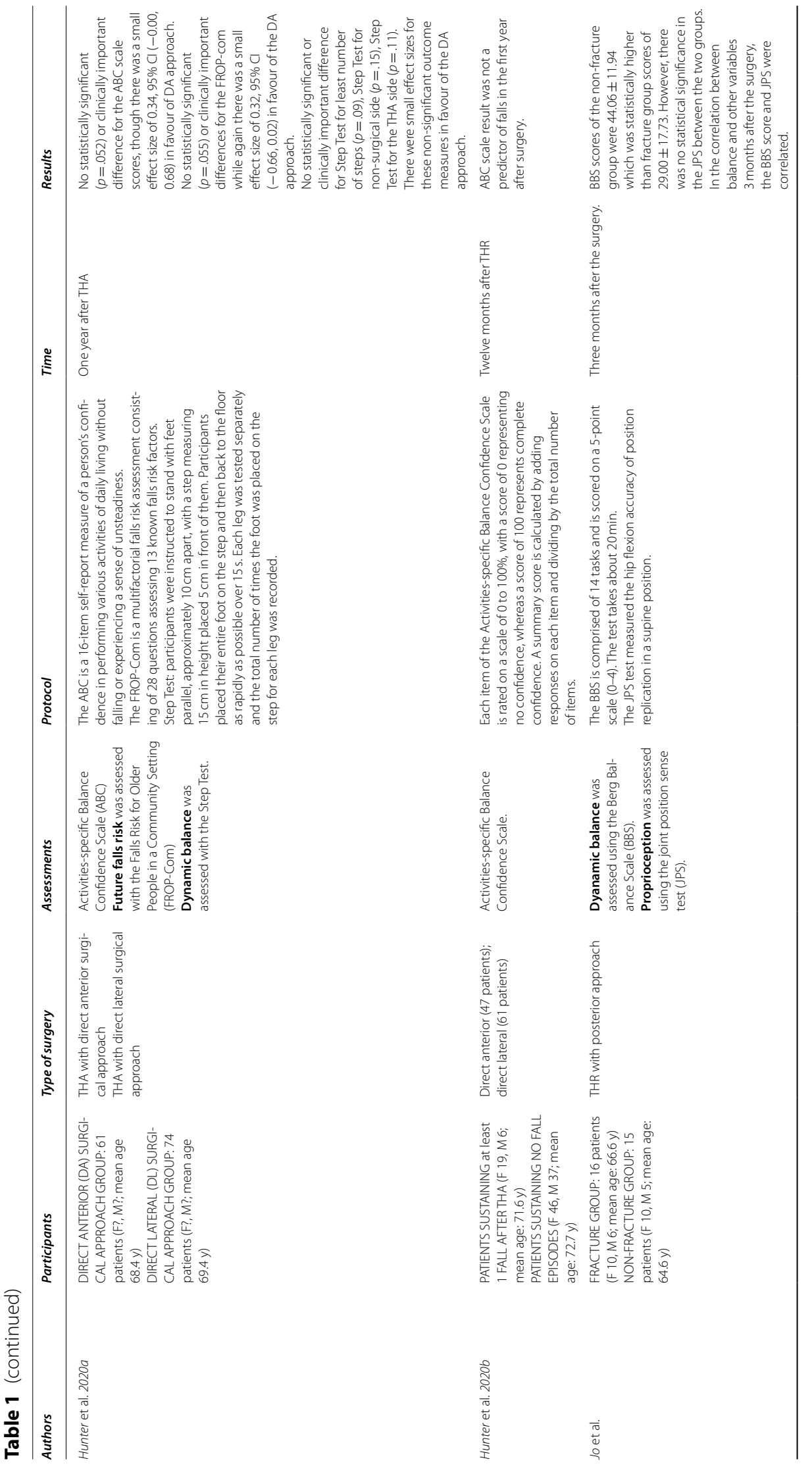




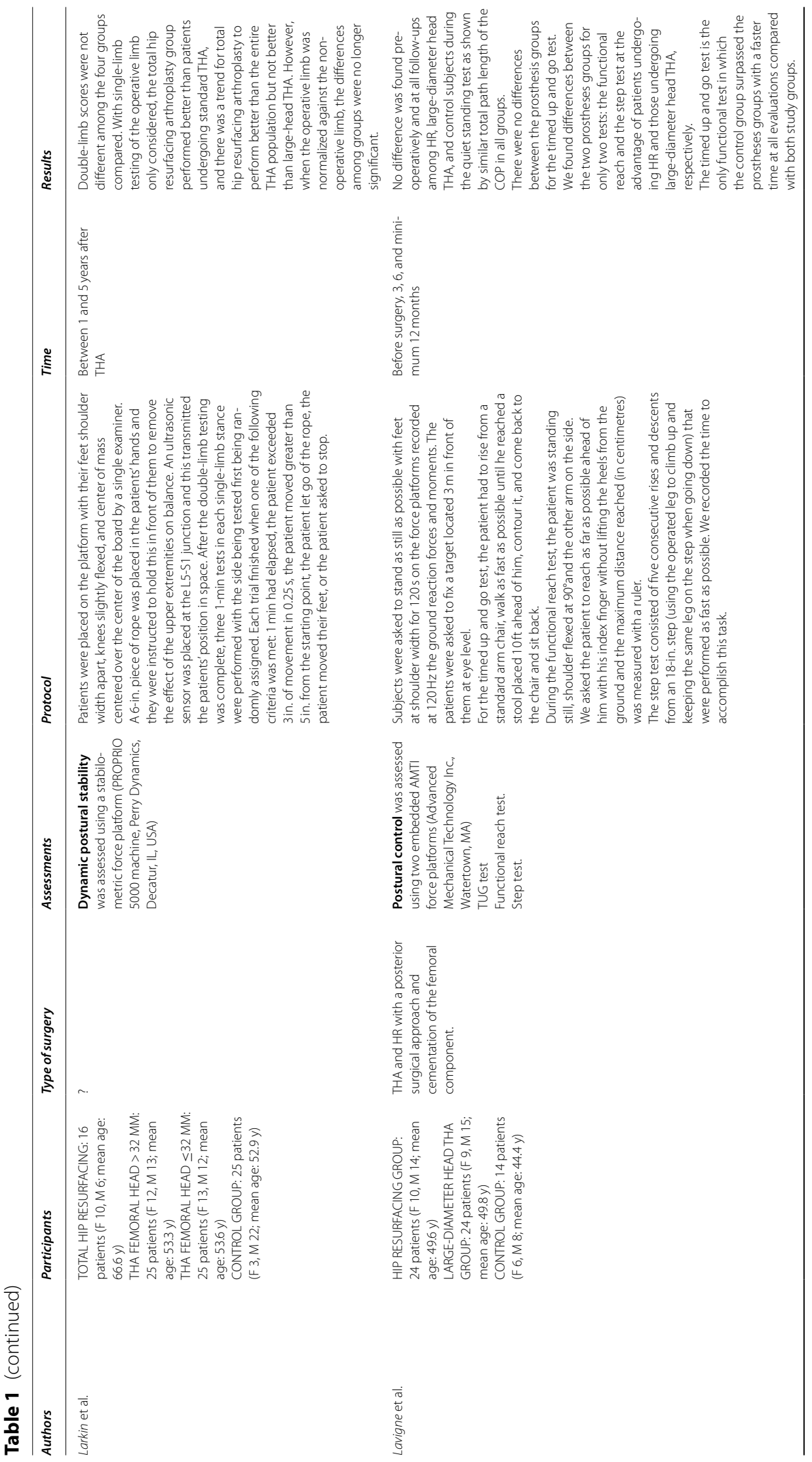




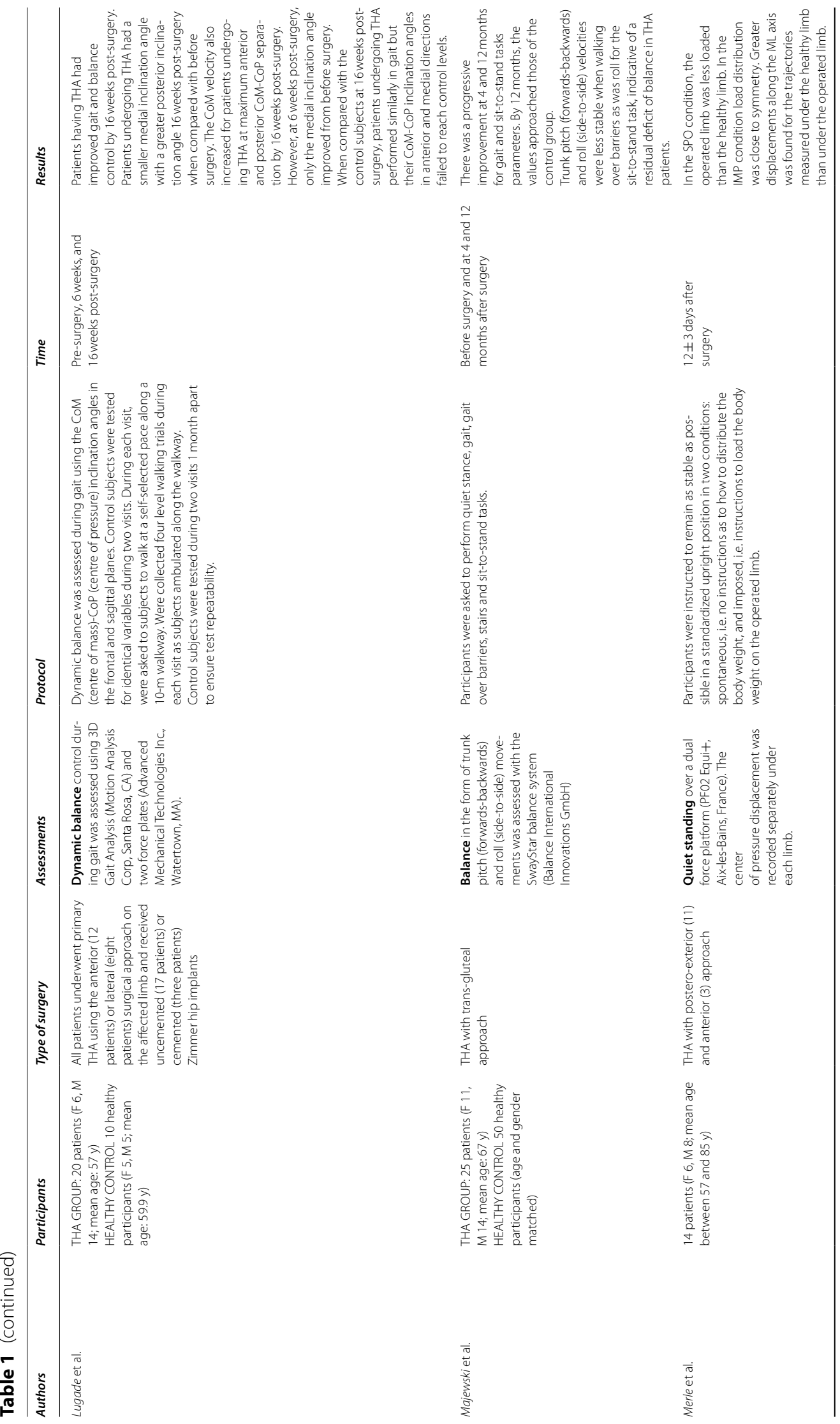




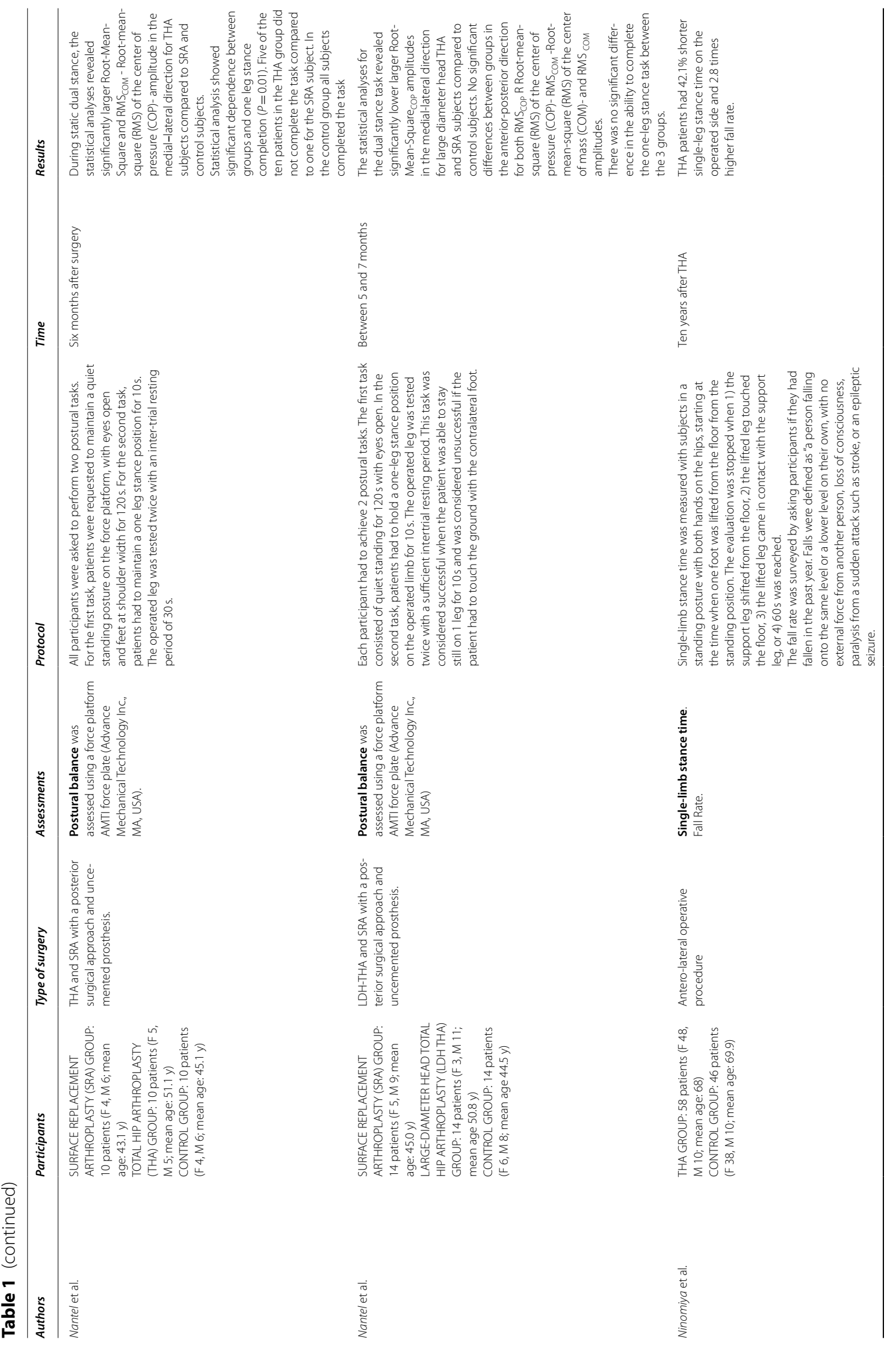




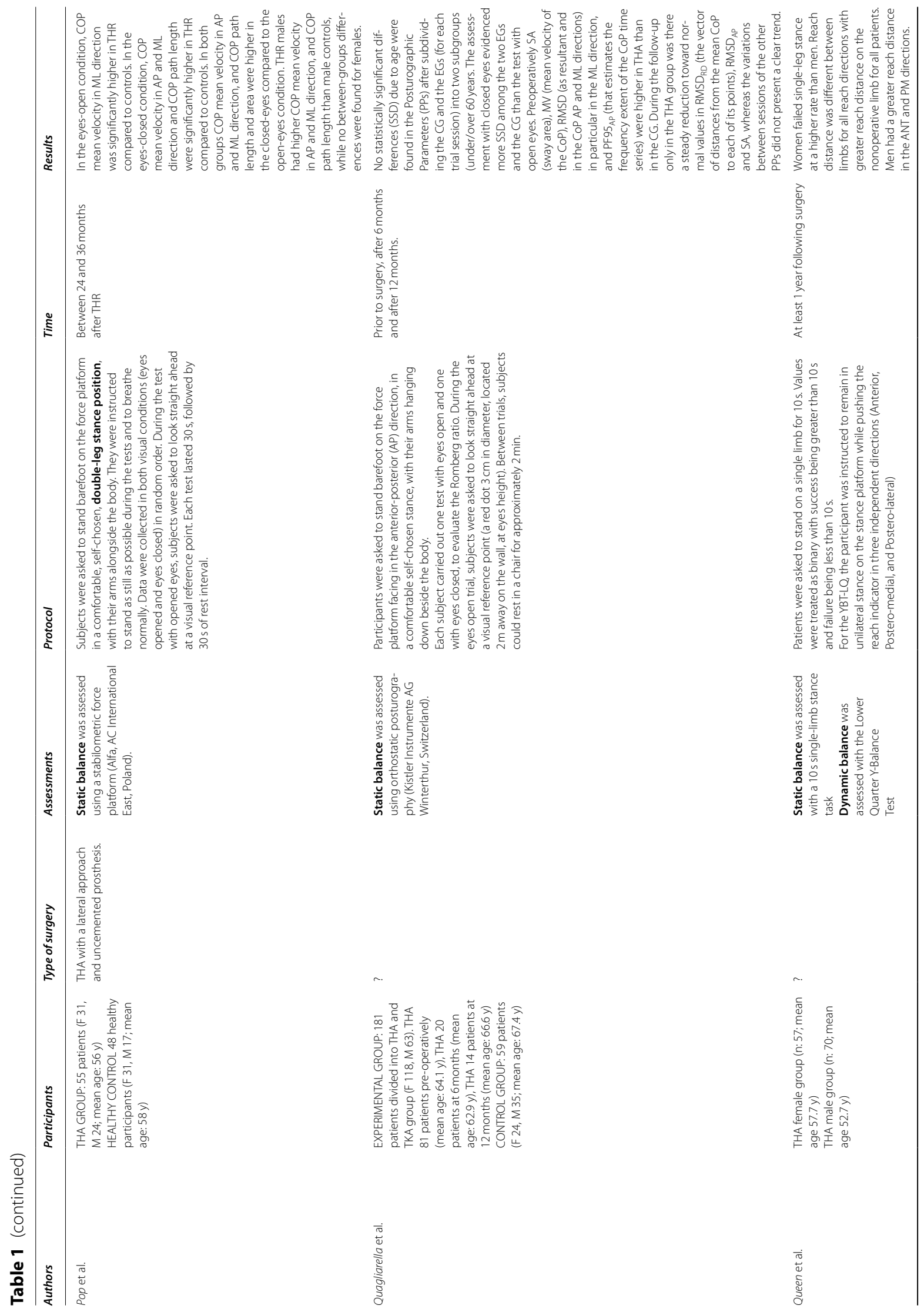




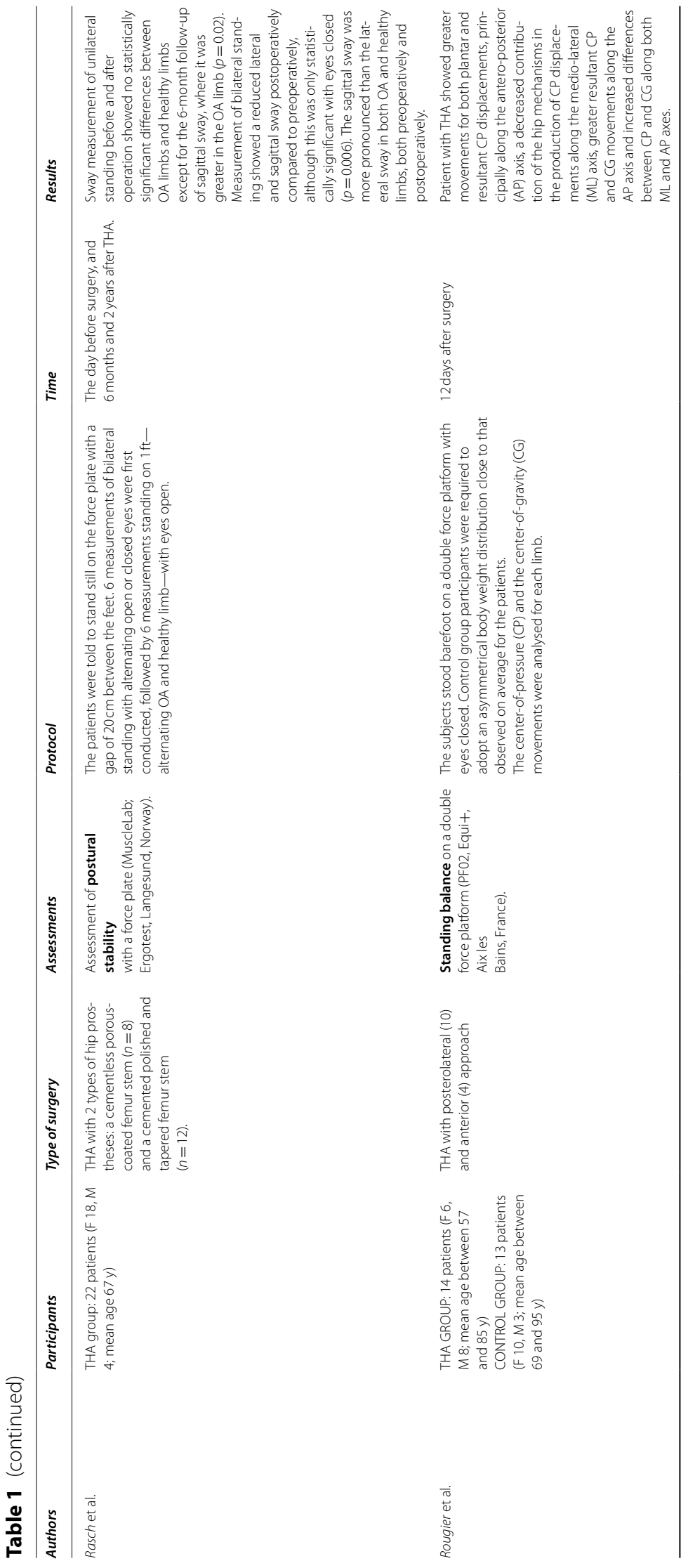


产

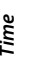

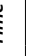

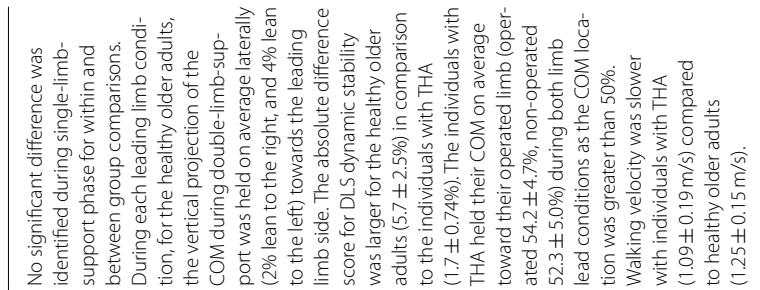

\section{흔

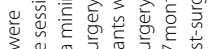

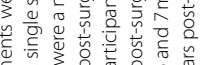

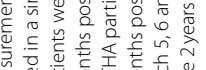

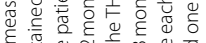

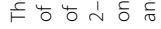

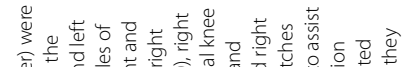

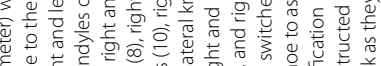

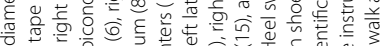

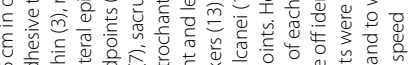

等

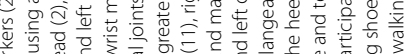

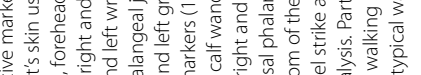

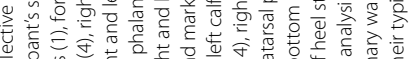

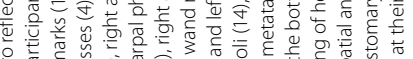

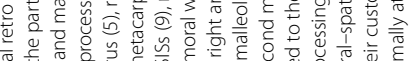

ఫัญ

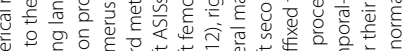

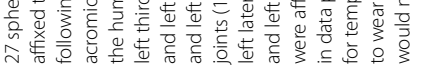

可

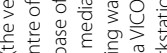

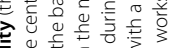

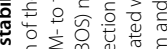

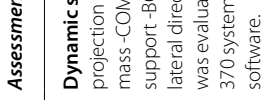

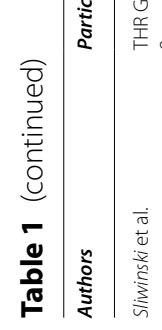

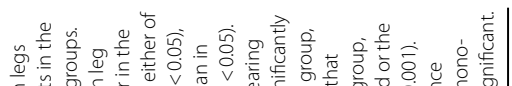

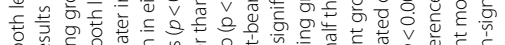

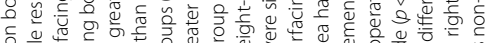

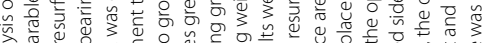

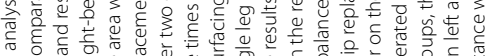

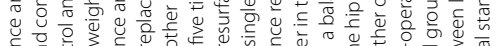

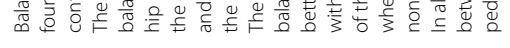

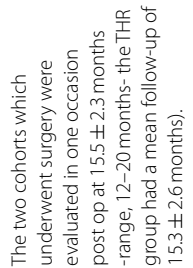

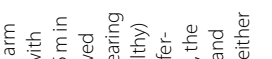

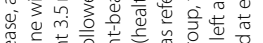
to

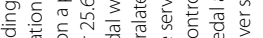
突

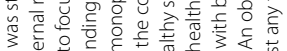

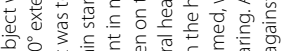
荺品

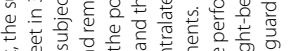

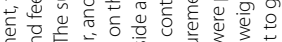

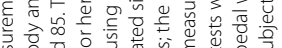

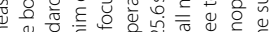

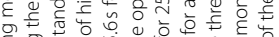

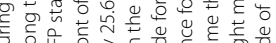

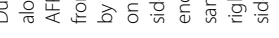

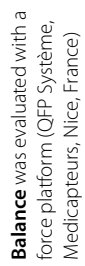

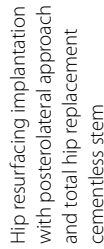

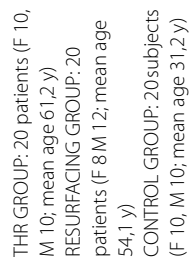

ज्ञ 


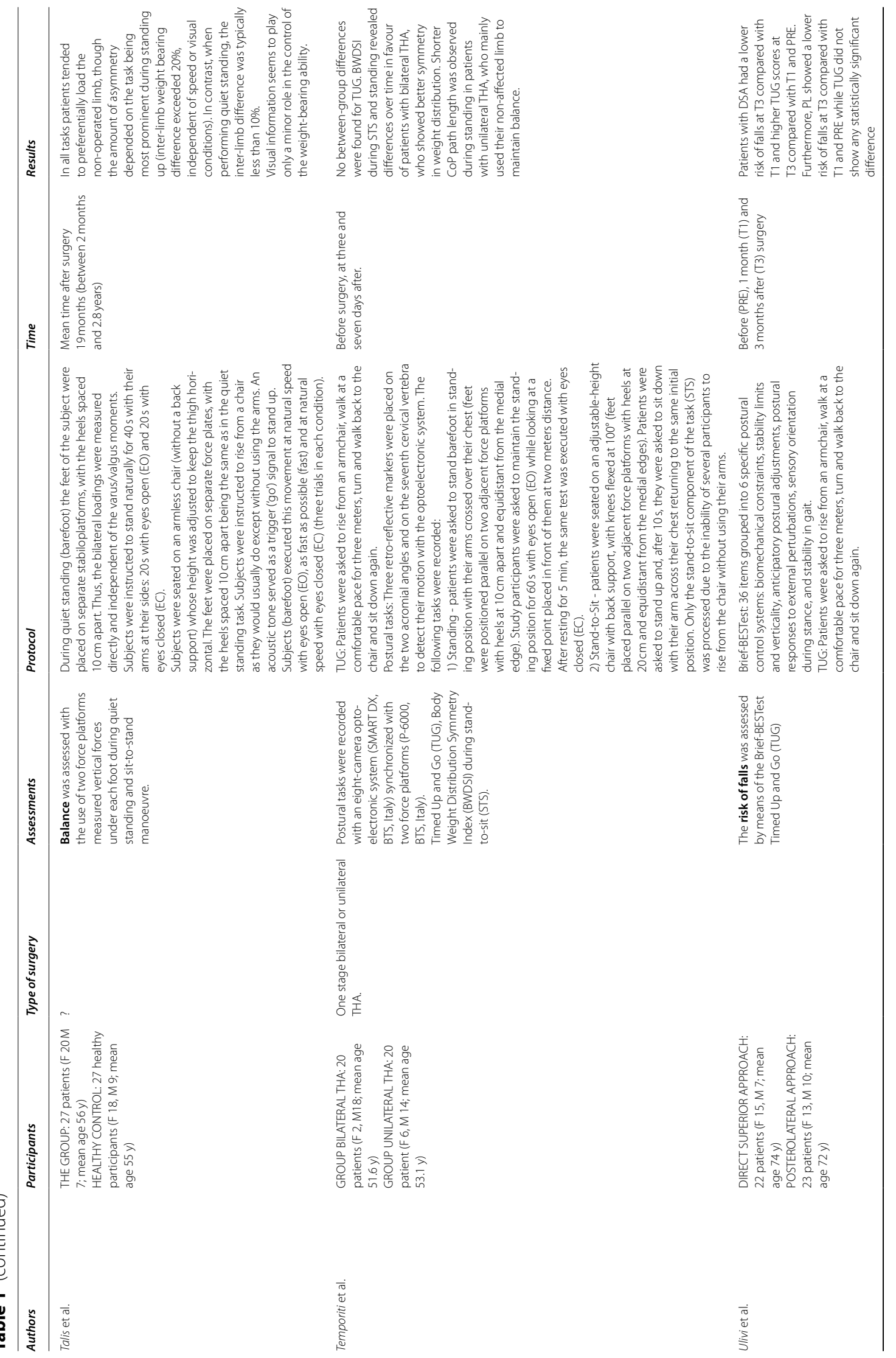




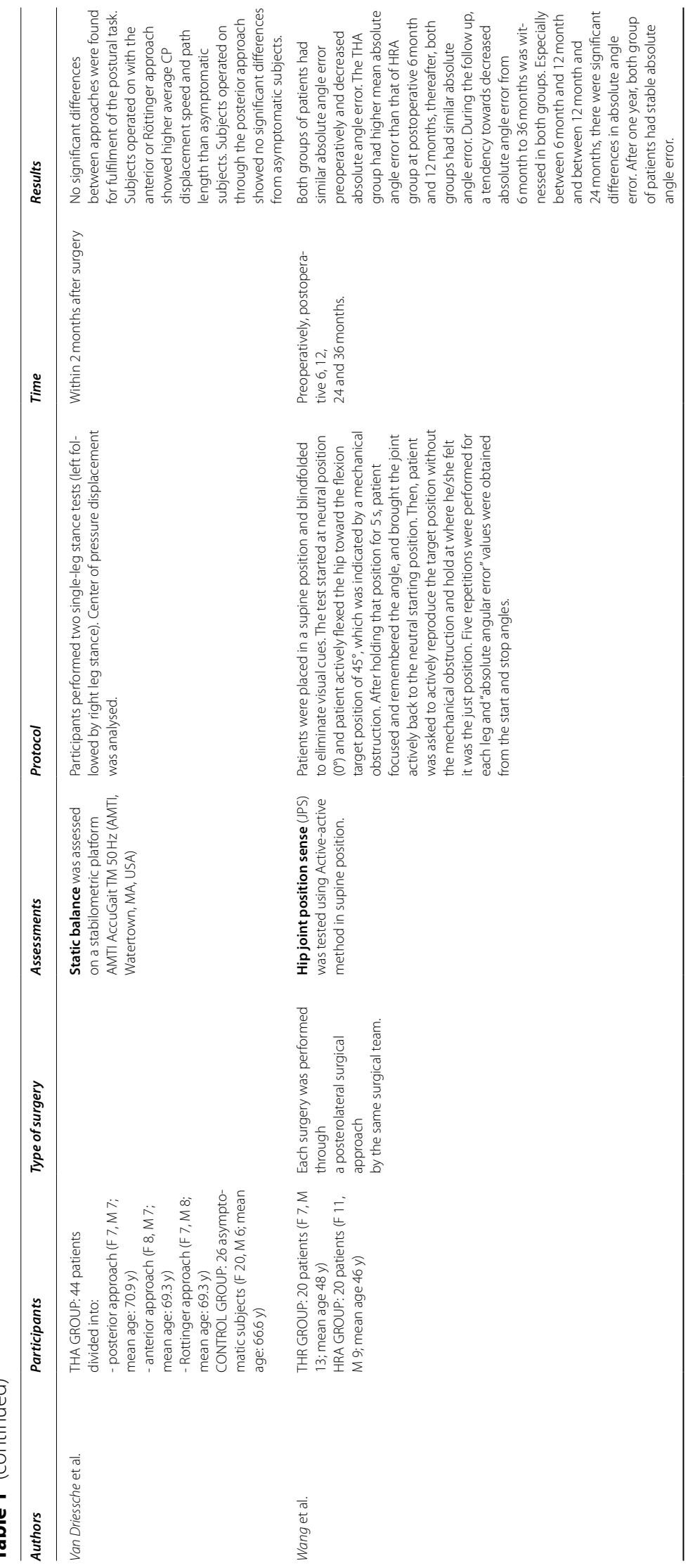




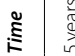

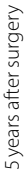

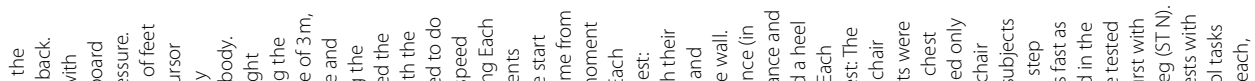

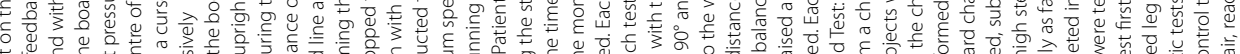

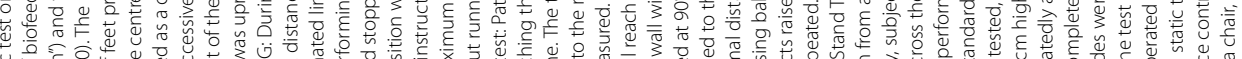

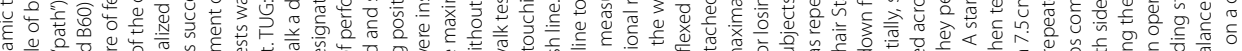

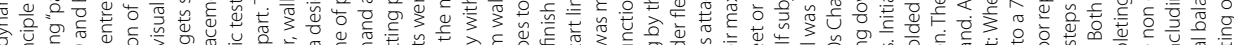

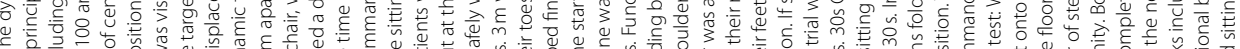
$\begin{array}{ll} & \\ & \end{array}$ 等

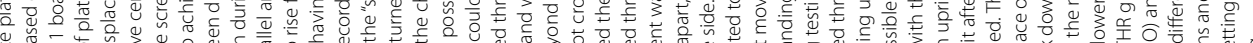
$\begin{array}{ll} & \\ 0 & \end{array}$

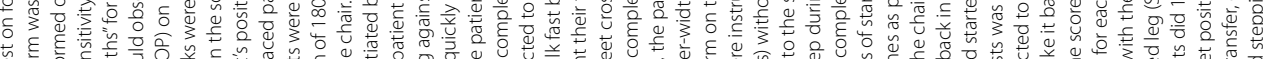

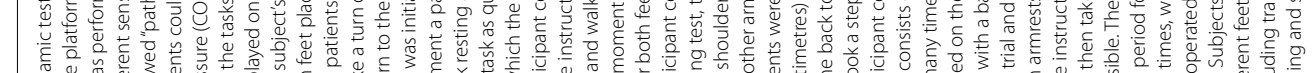

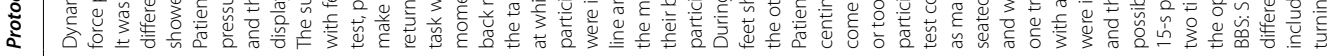

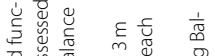

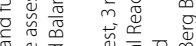

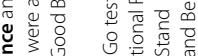

贾

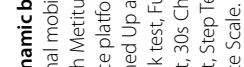

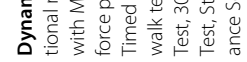

$\rightarrow$

胥

ฟั้े

紊贾

言

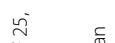

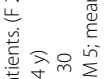

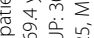

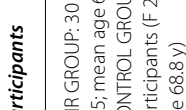

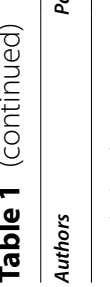

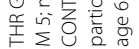

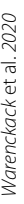




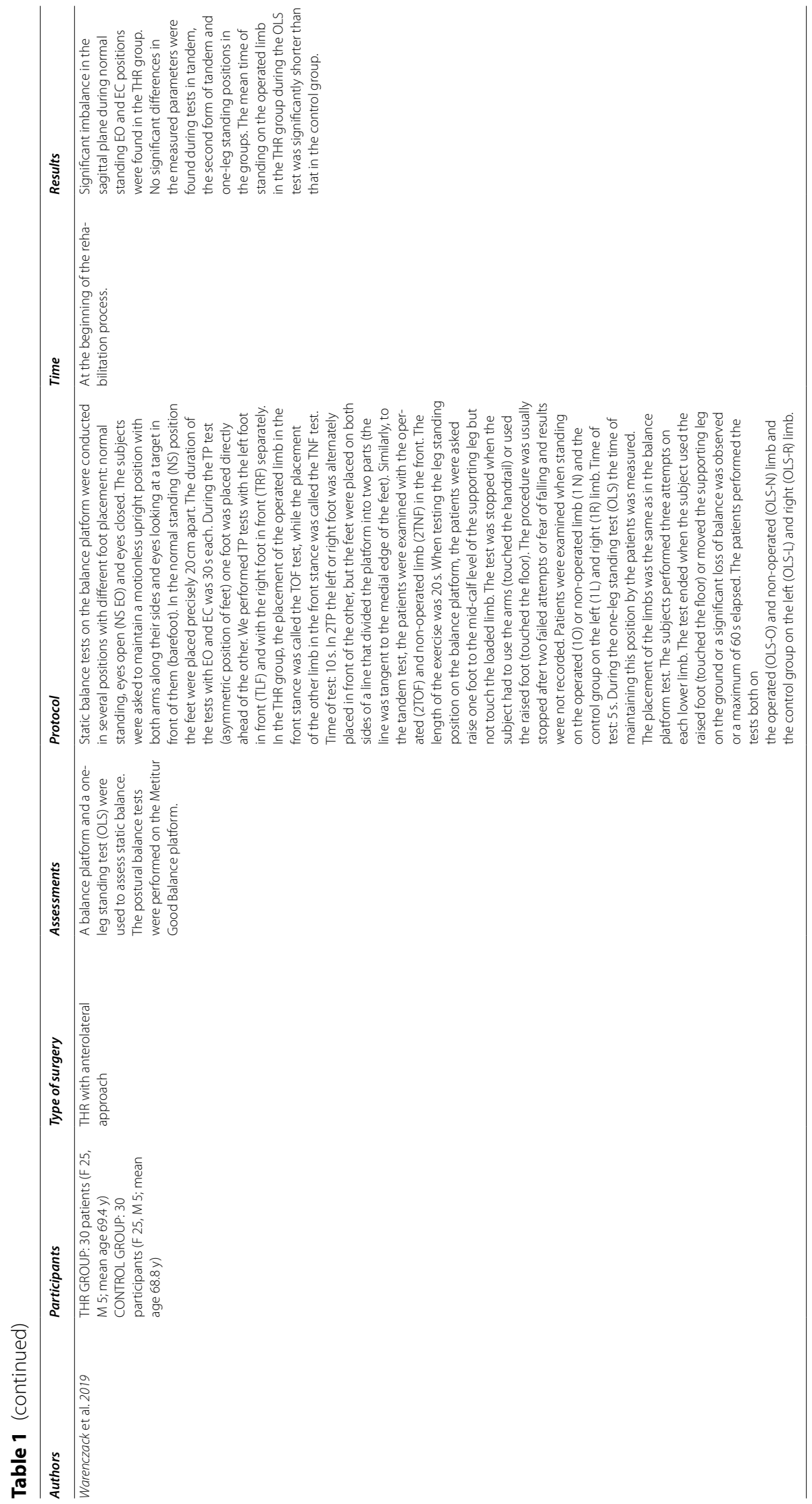




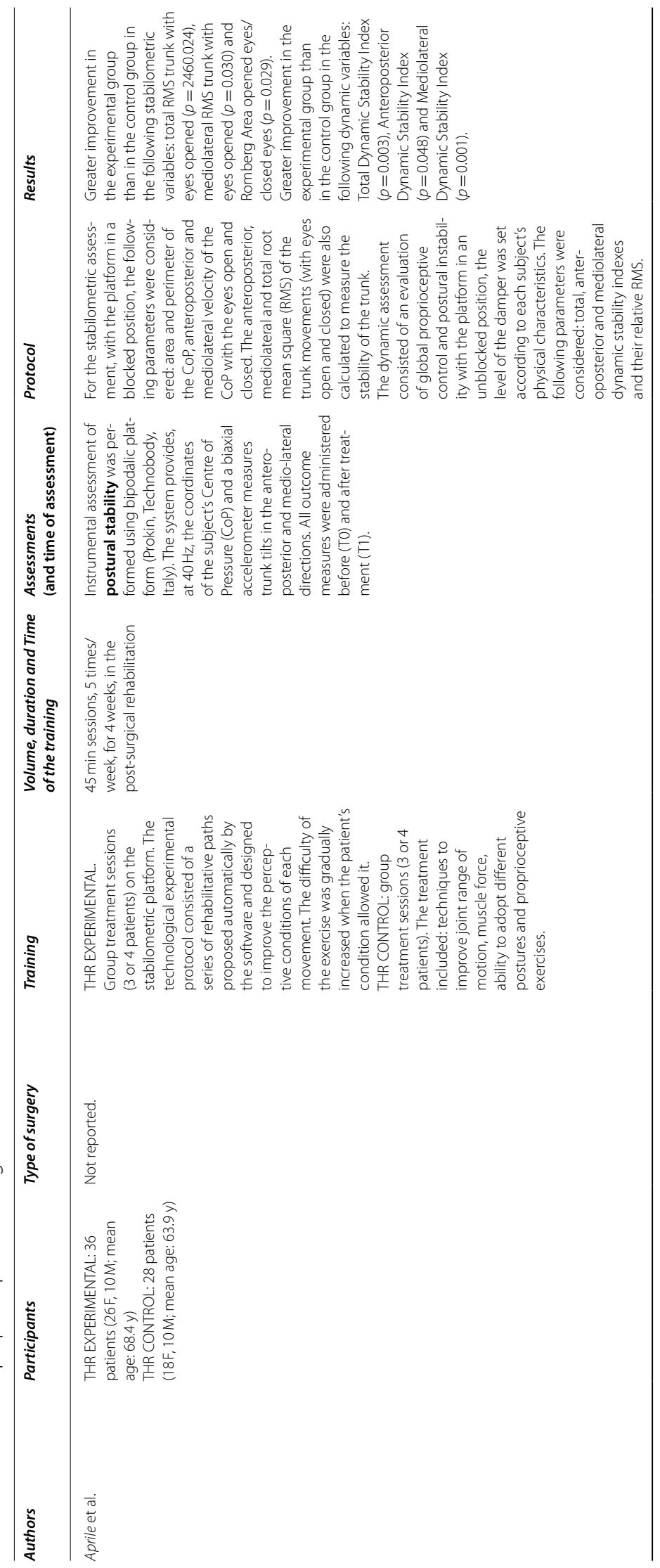




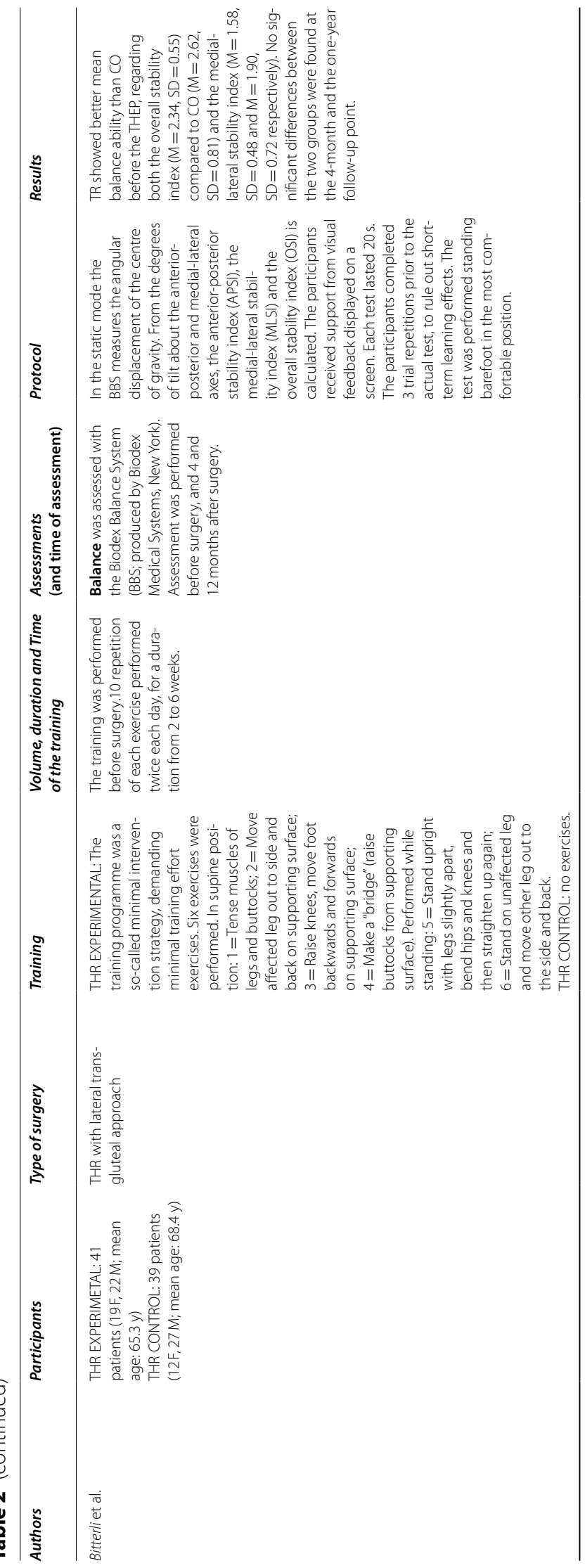




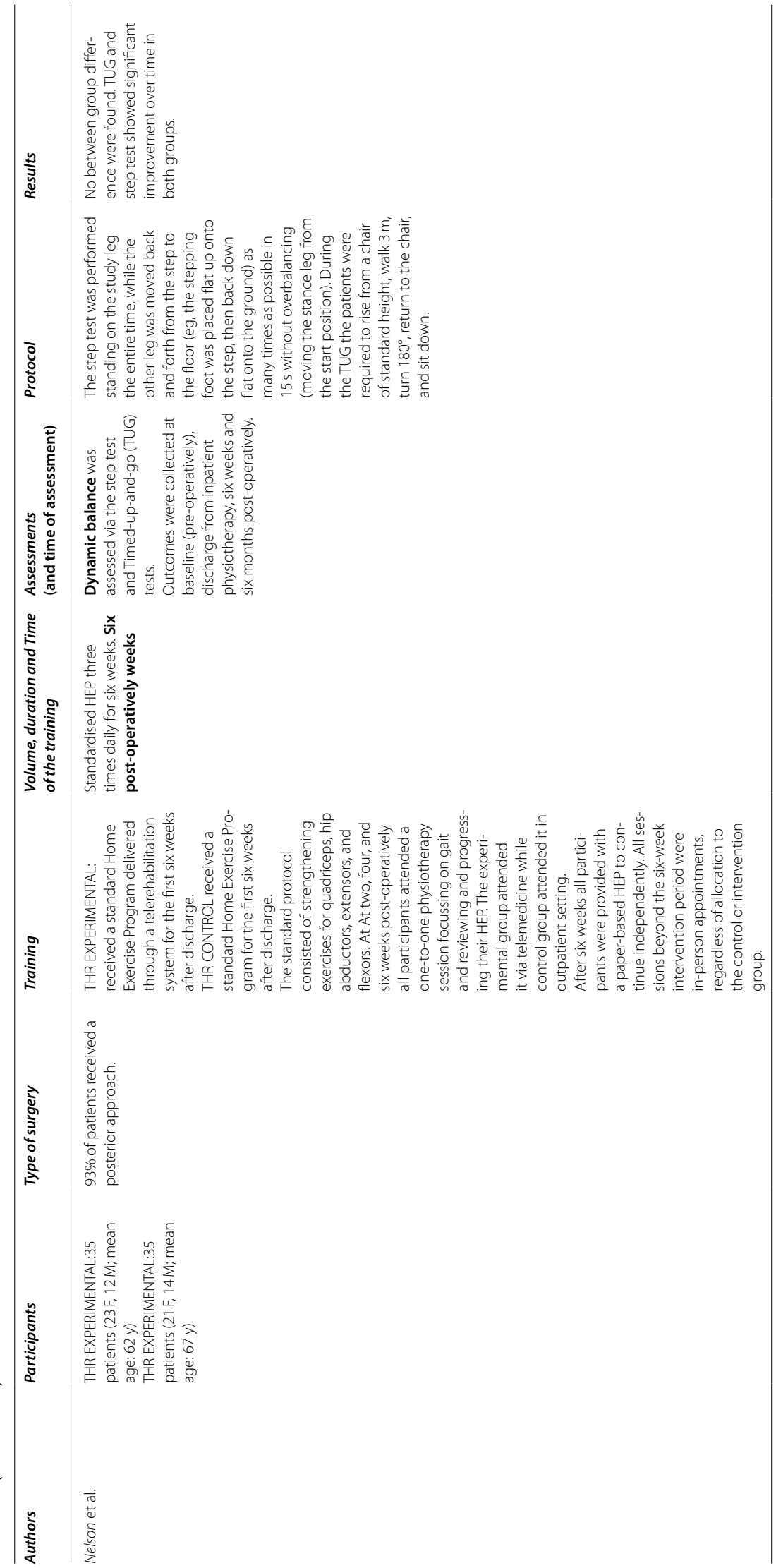




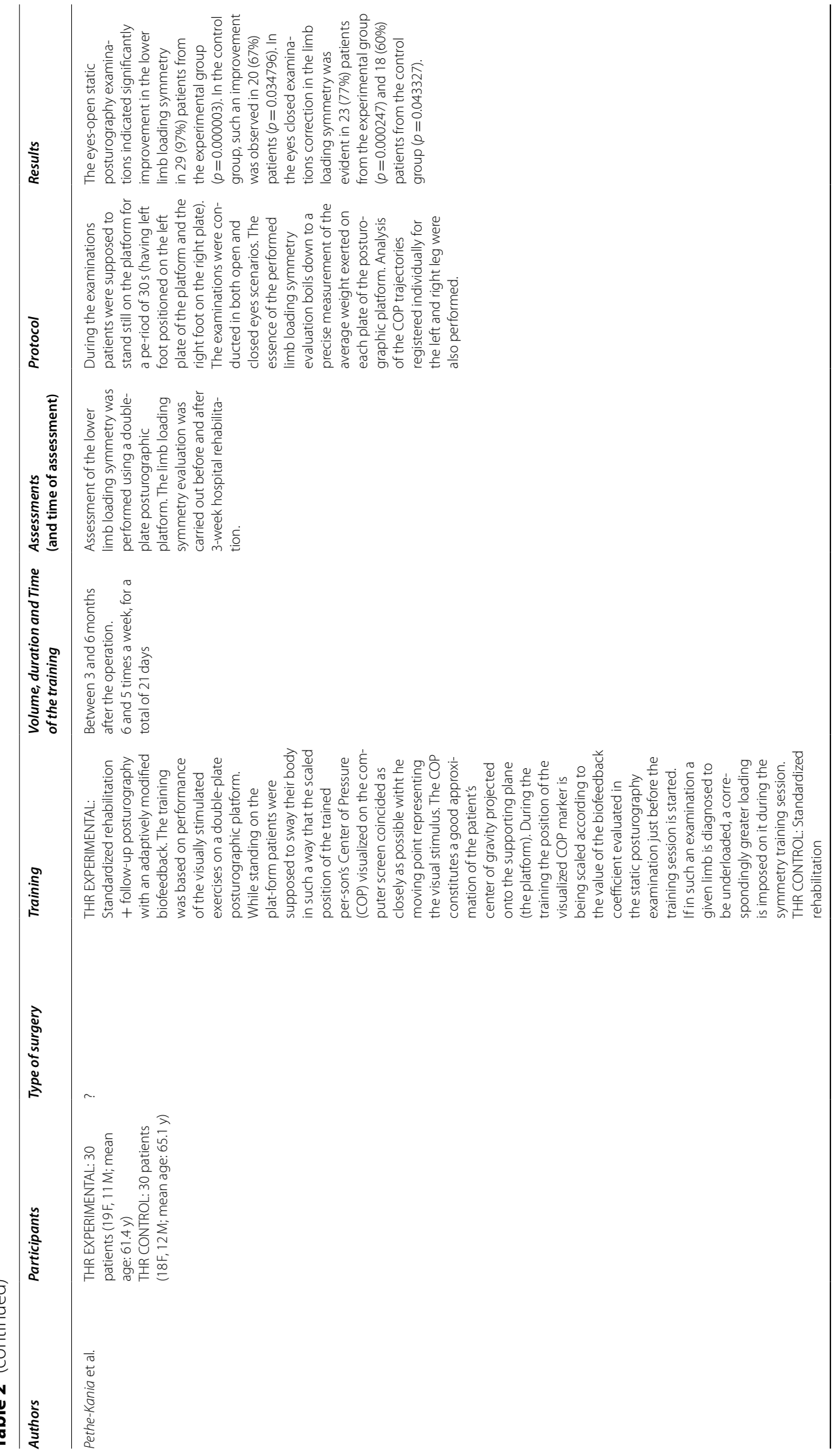




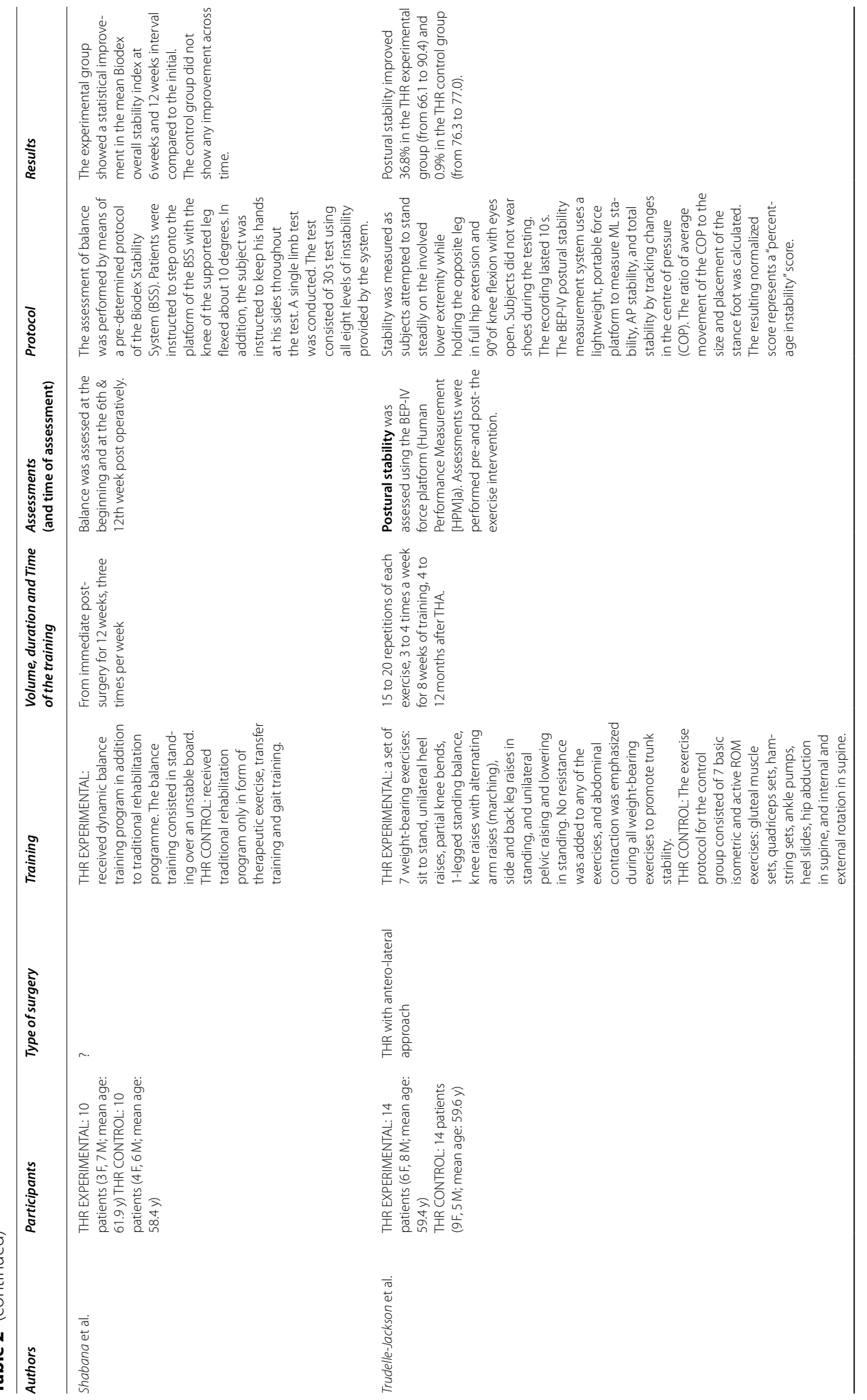




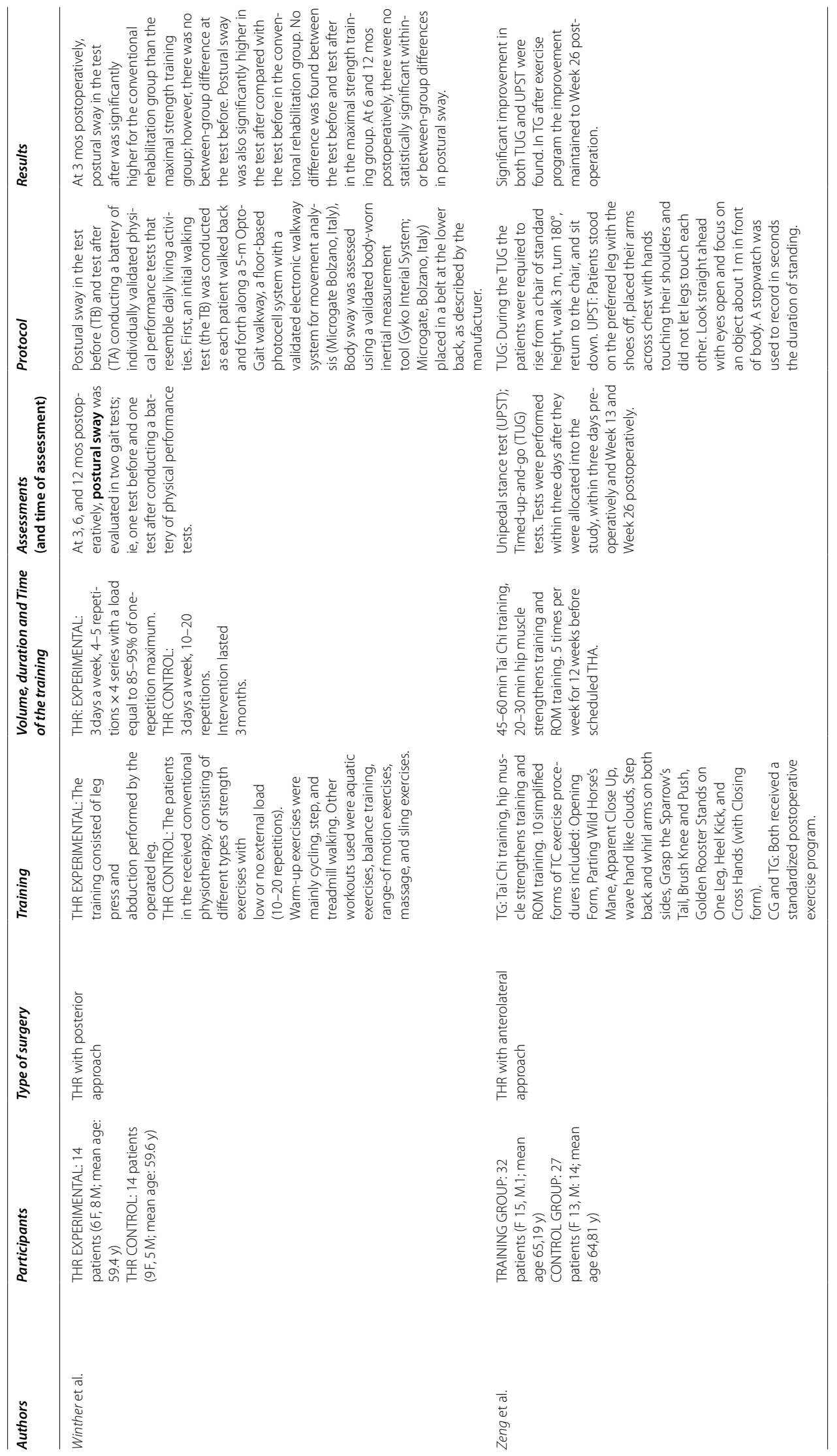




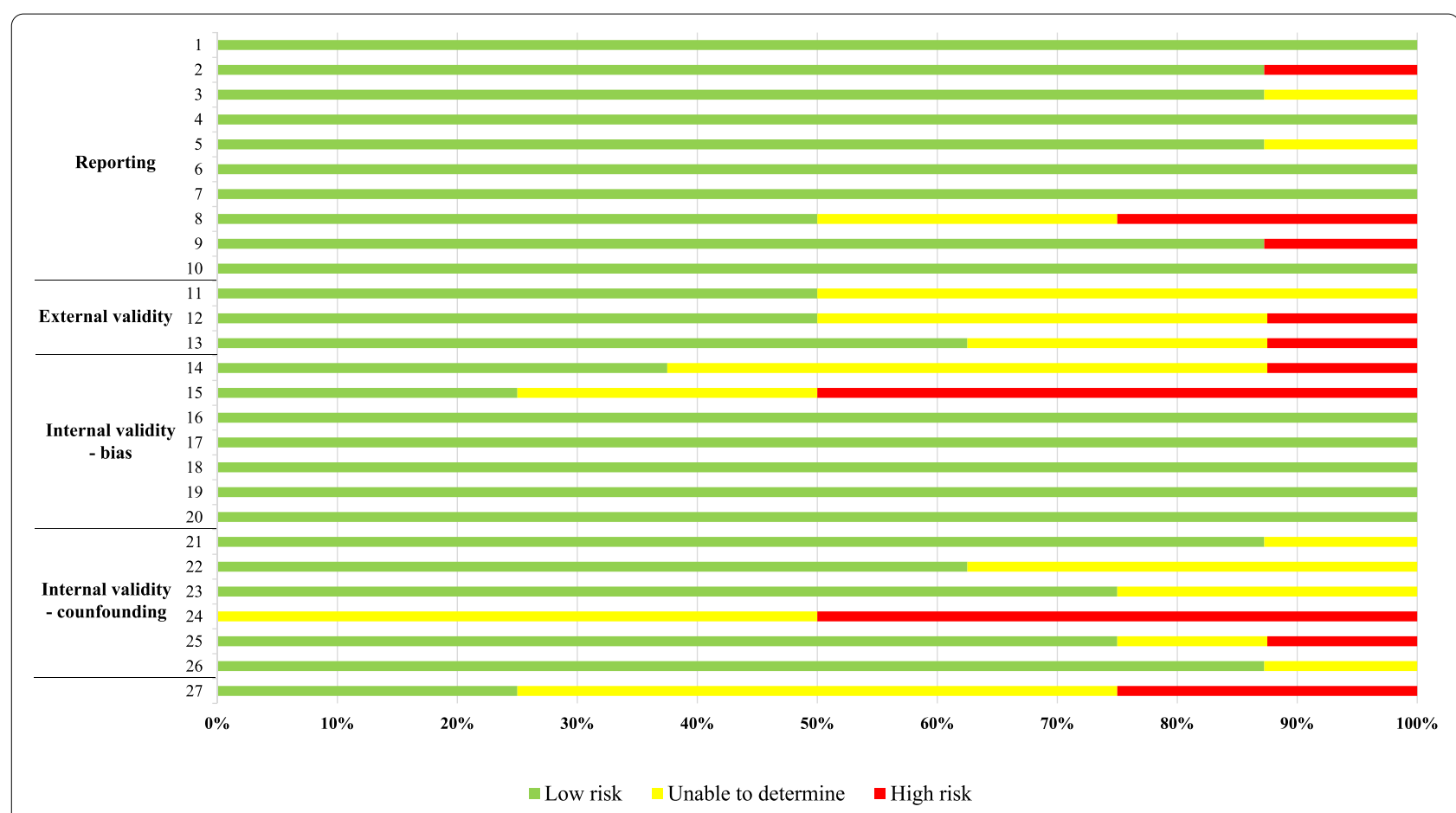

Fig. 2 Risk of bias across studies on balance and proprioception impairments and assessment, express as a percentage. Data are provided for each item of each domain of the modified Downs and Black checklist

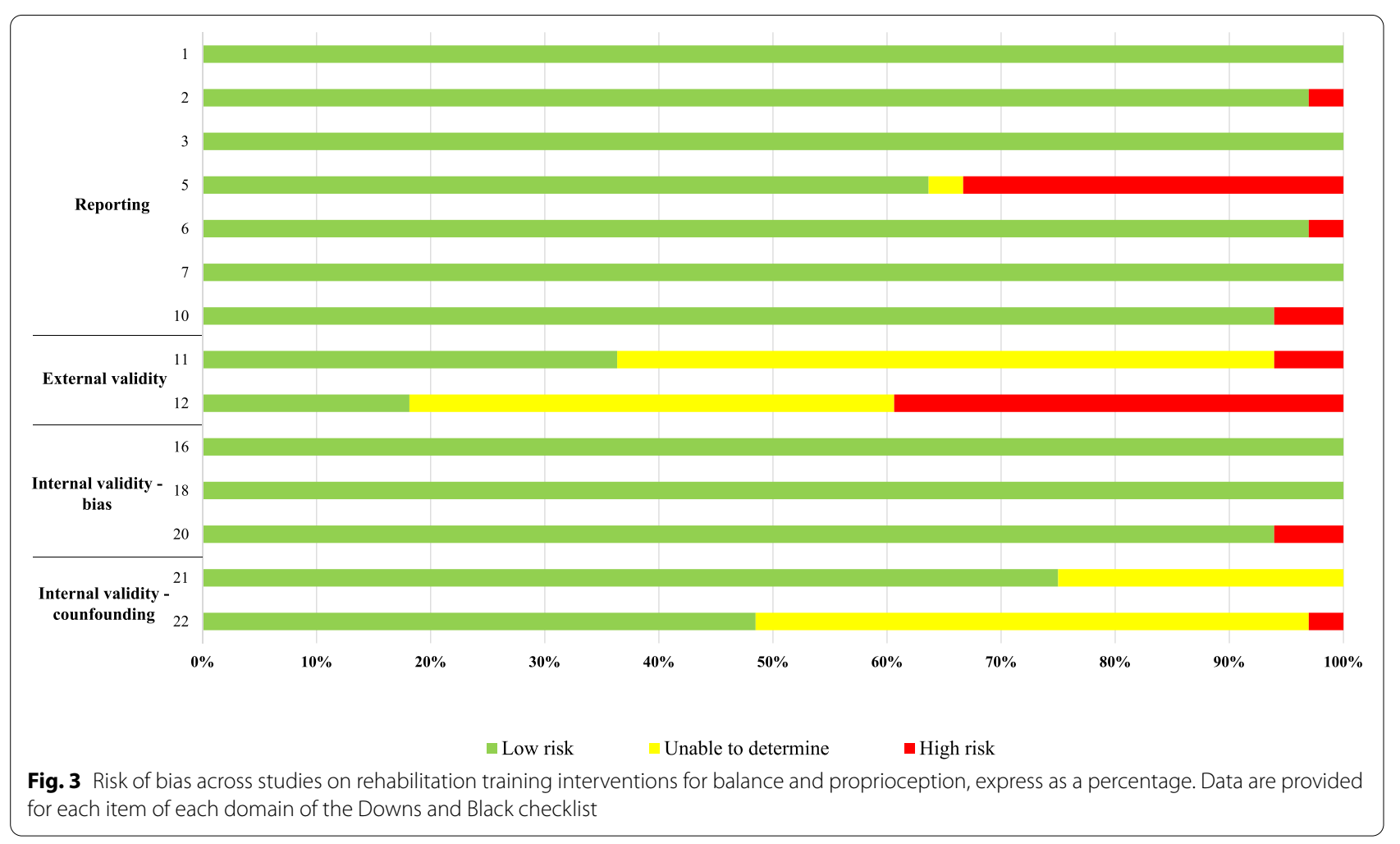


Regarding studies on balance and proprioception training, sources of high risk of bias were observed regarding the items 2, 8, 9, 15 and 24, i.e., the clear description of the outcome measures (reporting bias), at least an attempt to measure adverse events (reporting bias), the description of patients lost in follow-ups (reporting bias), blinding of the assessors (internal validity bias) and the concealment of the randomized assignment to study groups of both patients and health-care staff (selection bias), respectively. No information or no information mixed with sources of high risk of bias were observed regarding items 11, 12 and 13, which referred all to as external validity of the study (i.e., the representativity of the participants which were asked to participate in the study with respect to the whole population, the representativity of the participants recruited with respect to the whole population, and the representativity of the staff, places and facilities used for the study with regards to those usually used for patients), and the items 14, 22 and 27, i.e., the blinding of patients with respect to their own group assignment (internal validity bias), the recruitment of cases and controls in the same period of time (selection bias), and the statistical power of the study, respectively.

\section{Balance and proprioception impairment and assessment tools}

Static balance/postural stability was assessed in 20 studies [11, 35, 37-53, 63]; dynamic balance was assessed in 10 studies [34, 36, 41, 52, 54-59]; proprioception in terms of sense of joint position was assessed in 2 studies [60, 61]; clinical scales and other test batteries for balance assessment were used in 10 studies [11, 13, 40, 41, $52,59,60,62-64]$.

\section{Static balance / postural stability}

Static balance, also referred to as postural stability, was assessed by double- or single-limb stance tasks on force platforms in 11 studies, which analysed centre of pressure (COP) velocity and displacement [11, 38-47]. Four of these $[41-43,47]$ were performed in three groups: patients with THA, patients with Hip Resurfacing (HR), and healthy age-matched controls. Two studies were performed on patients undergoing a regular size THA, and these reported a higher COP displacement in patients with THA compared to HR and healthy agematched controls during the double-limb standing task $[42,47]$. The other two studies which involved patients with large-diameter THA reported no differences in COP path length and displacement between controls, HR and THA patients [41], or a lower medial-lateral displacement in HR and THA compared to controls [43]. A better balance performance in HR and controls compared to THA was found also during the singlelimb stance task [42, 47], and no differences between the three groups were found in the study involving patients with large-diameter THA [43]. Timing of assessment was heterogenous between studies, and ranged between 5 - and 15-months following surgery. One study reported an increased COP displacement in THA patients 12 days after surgery in comparison to healthy matched controls [38]. The last study [39] compared 3 groups of patients within 2 months after surgery, who received 3 different surgical approaches (posterior, anterior and Röttinger approches) and a group of healthy matched controls. No significant differences between approaches were found for fulfilment of the single-limb balance tasks. However, subjects operated on with the anterior or Röttinger approach showed higher average COP displacement speed and path length than controls. Subjects operated on through the posterior approach showed no significant differences from controls.

Two studies compared COP displacement during double-limb stance, in open- and closed-eyes condition $[44,45]$. Both studies reported in the closed-eyes condition a higher displacement of COP in THR compared to healthy age-matched controls, at 6- and 12-months post-surgery, and in a single assessment between 24- and 36-months post-surgery [44]. A significant improvement was reported between 6- and 12-months following surgery [45].

A lower displacement of the COP was reported in patients undergoing unilateral THA in comparison with patients undergoing bilateral THA [11]. Two studies having no control group reported a reduction of postural sway in the post-operative compared to pre-operative period during double-limb stance, and a higher sagittal sway compared to medial-lateral sway during single-limb or tandem stances, independently from the examined $\operatorname{limb}[40,46]$.

One study assessed stability by means of pre-determined protocols of an instrumented platform (Biodex Balance System), and it reported an improvement in the parameters related to postural stability at 6 months after surgery compared to pre-surgery data [48]. Another study, using another instrumented device (PROPRIO 5000 machine) found that with single-limb testing of the operated limb, the HR group performed better than patients undergoing standard THA, and there was a trend for HRs to perform better than the overall THA population, but not better than large-headed THAs [49]. In the same study [49], no differences were found for postural stability in double-limb stance between patients with HR, patients with THA femoral head $>32 \mathrm{~mm}$, patients with THA femoral head $\leq 32 \mathrm{~mm}$, and the control group. 
Four studies assessed the time that patients were able to spend in a single-limb stance position [ 50-52]. Two of them found a significantly shorter time in THA group compared to healthy controls for the operated side [ $51,52]$, while another study without a control group of healthy participants, reported a shorter time in the operated compared to the non-operated side [50]. The fourth study reported a higher failure rate during the stance position in female patients compared to male patients, more than 1 year after surgery [63].

Two studies assessed the between-limbs loading difference while standing $[11,53]$. A higher loading of the non-operated limb was reported in patients with unilateral THA $[11,53]$, while loading was more symmetrical in patients with bilateral THA [11].

In the study by Merle et al. [37], THA patients were asked to stand on two force plates in two conditions: a comfortable position, or with the requirement to load the operated limb. In the first condition, the operated limb was less loaded than the healthy limb, while in the second condition load distribution was close to symmetry, but a higher displacement along the medial-lateral axis was found for the trajectories measured under the healthy limb than under the operated limb.

Finally, balance in terms of COP displacement seems to be not affected by the position of the elbow (straight or flexed) for holding a crutch at a mean of 4 days after surgery, even if the elbow flexed increases shoulder loading [35].

\section{Dynamic balance}

Two studies investigated dynamic balance during the performance of a 5-times-sit-to-stand [54] and a 5-timessquat [55] on force plates. Within 1 month after surgery, THA patients showed a higher asymmetrical loading and higher anterior-posterior (AP) and medial-lateral (ML) COP displacement during squatting movements when compared with healthy age-matched controls [55]. Asymmetry and COP displacement were found to be significantly different from healthy controls 1 year after surgery during sit-to-stands movements [54].

Two studies investigated dynamic balance during walking by means of force platform and 3D motion analysis $[56,57]$. One study reported that, compared with healthy age-matched control subjects, THA patients showed greater frontal plane (FP) center of mass-center of pressure (COM-COP) inclination angles and smaller sagittal plane angles which improved postoperatively, but remained significantly different from healthy controls [57]. The other study [56] found no differences during single-limb-support phase for within and between group comparisons for the COM projection over the base of support. During the double-limb support phase for the healthy age-matched older adults serving as a control group, the vertical projection of the COM during doublelimb-support was held on average laterally towards the leading limb side, while the individuals with THA held their COM on average toward their operated limb both limb lead conditions.

One study investigated dynamic balance in response to a sudden medial-lateral perturbation in three groups of THA patients undergoing the three different surgical approaches [58]. It was found that, in the case of directlateral and antero-lateral exposure, Lehr's damping ratio significantly decreased compared to the preoperative values at 6 weeks postoperatively, but it increased steadily afterwards. Lehr's damping ratio while standing on the affected limb was significantly lower - even at 6 months postoperatively - than that of the healthy age-matched control group. In the case of posterior surgical approach, Lehr's damping ratio continuously increased in the postoperative period and corresponded to that of the control group at 6 months after THA.

Balance in response to sudden platform inclinations and translations was investigate also in another study [34], which found no differences between THA patients 4 months after surgery and healthy age-matched controls.

Three studies investigated dynamic balance during a step test. One year after THA no differences for least number of steps, Step Test for non-surgical side and Step Test for the THA side, between patients operated with lateral approach and patients operated by the anterior approach were found [59]. The other study [41] reported that patient operated with the large-diameter head THA were able to complete the task approximately $3 \mathrm{~s}$ faster than the HR group $(p=0.001)$. A few numbers of steps were reported in THA patients 5 years after surgery [52].

One study investigated dynamic balance in the immediate post-surgical period during a biofeedback test based on COP displacement, and found that in THA patients Time and distance of COP displacement were higher, the performance of the task was slower and the displacement of the COP was higher compared to controls [52].

The last study [36], investigated postural sway by means of a portable system embedded with gyroscopes, applied over the low back, during the performance of gait and gait over barriers, stairs climbing and sist-to-stand tasks. It was found a progressive improvement of balance between 4 and 12 months after surgery for gait and sitto-stand tasks parameters. At 12 months post-surgery, THA patients results approached those of healthy control group participants, however, trunk pitch (forwards-backwards) and roll (side-to-side) velocities were less stable when walking over barriers as was roll for the sit-to-stand task, indicative of a residual deficit of balance in THA patients. 


\section{Hip joint position sense}

The study by Jo et al. [60] investigating proprioception by means of hip joint position sense reported no differences between patients undergoing THA for osteoarthritis or after hip fracture 3 months after surgery, while Wang et al. [61] reported that THA patients had higher mean absolute angle error than HR patients at 6 and 12 months from surgery; after 1 year and up to 36 months, both groups had similar absolute angle error. In either study, results were not compared with a control group of healthy age-matched participants.

\section{Clinical scales and other tasks}

Berg Balance Scale was used in three studies. Warenczackc et al. [52] reported no differences between THA patients and healthy age-matched controls at 5 years after surgery. Chang et al. [40] found that Berg balance test decreased significantly after 2 weeks from THA surgery and improved gradually thereafter, reaching the highest score at 6 months. Jo et al. [60] reported that 3 months after surgery BBS scores of patients undergoing THA for degenerative arthritis were significantly higher than patients undergoing THA because of fractures scores.

Activities-specific Balance Confidence Scale (ABC) was used in two studies $[13,59]$. No differences were found at 1 year from surgery when patients operated on by a direct anterior and direct lateral approaches were compared [59]. Moreover, ABC scale was found not to be a predictor of falls in THA patients [13].

Timed-up-and-go was used in four studies. No differences have been reported between patients with unilateral and bilateral THA [11], patients undergoing either a direct superior approach or a posterolateral approach for surgery [64], as well as in patients undergoing largediameter THA or HR. [41] However, THA patients were significantly slower than healthy age-matched controls [ 41, 62].

Functional reach test was used in three studies [ 40, 41, 62]. Patients with THA reached shorter distance than healthy age-matched controls when studied between 3 and 5 years after surgery [ 41, 62]. Accordingly, another study reported no significant improvements between 2 weeks and 1 year after surgery [40]. Patients undergoing HR had better performance from 3 to 12 months after surgery when compared to large-diameter heads THA patients [41].

One study reported a higher reaching distance during the Low Quarter Y-balance test in male compared to female patients, more than 1 year after surgery [63].

Risk of falls was assessed in two studies. The first study the assessment was performed by means of the Falls Risk for Older People in a Community Setting (FROP-Com) tool; the authors found no differences between patients undergoing THA with the direct anterior approach and patients undergoing THA with the direct lateral approach [59]. The second study, performed the assessment by means of the Brief BEST-test in two groups of THA patients, undergoing either a direct superior approach or a posterolateral approach for surgery [64]. A reduction in the risk of falls was observed in both groups between 1- and 3-months following surgery.

Fall rate was assessed in one study, which reported a 2.8 times higher fall rate in THA patients compared to healthy age-matched controls in the ten years after surgery [51].

The 3-m walk and 30-s chair standing tests were used by Wareczack et al. [62] 5years after THA surgery. Patients with THA performed shorter distance and a lower number of repetitions, respectively, when compared to healthy age-matched controls.

\section{Balance rehabilitation training}

Three studies investigated the effects of a training intervention in the immediate post-surgical rehabilitation [65, $66,72]$, one study in the period between 4 and 12 months after surgery [67], and one study in the period between 3 and 6 months after surgery [70]. Four studies reported a higher increase in balance in patients undergoing balance training $[65,66,70,72]$, while the study by Nelson et al. [66] reported a similar between-groups increase in balance following a non-specific balance training.

Two studies investigated the effects of a pre-operative balance training program on the post-operative outcomes. One study found positive effects of the preoperative balance training, based on Tai-Chi practice performed up to week 26 post-surgery [68], while another study found no differences between the groups in the post-surgery [69].

Better balance abilities while walking, in terms of reduced postural sway, were found in a group of THA patients undergoing a strength training protocol in comparison with THA patients undergoing a traditional rehabilitation [71]. This result was observed after a fatiguing test battery 3 months after surgery, while no betweengroup differences were observed at 6 and 12 months after surgery.

All the studies referred to balance or postural stability. The term proprioception was not used.

\section{Discussion}

\section{Balance and proprioception impairment and assessment tools}

The first aim of this review was to investigate if patients following THA show impairments in balance and proprioception. The high heterogeneity of studies 
methodologies and the lack of controls groups of healthy participants, or comparisons with normative data, makes difficult drawing conclusions. However, almost all the studies comparing results of THA patients with healthy controls reports significant differences between-groups, with THA patients have worse balance performance than healthy controls. Impairments have been reported during static $[38,39,41,44,47,51,63]$ and dynamic balance assessments $[36,54,58]$, as well as with the assessment by means of clinical scales or tests batteries [41, 51 , 62-64].Only 4 studies reported no differences between THA patients and controls in balance during the singlelimb phase of walking [56], the Berg-balance scale results 5 year after surgery [62], and in response to balance perturbations [34, 58]. Further studies with control groups of healthy participants are needed to better clarify these results.

Regarding proprioception impairments, it should be mentioned that in the only two studies retrieved for this review [60,61], the results of patients with THA were not compared to results of a control group. Further studies are strongly recommended given the essential role of proprioception for both static and dynamic balance performance. It is not possible to draw conclusion on the timing of rehabilitation in which impairments are higher. It seems that a general improvement is reported in the post-operative compared to the pre-operative period [ 40 , $46,48]$. Some studies with more than one assessment in the post-surgical follow-up reported a balance improvement across time $[45,58,64]$, while other did not $[40,46$, 49]. However, there are many studies reporting balance impairments also years after surgery $[40,41,45,51,54$, $62,63]$, and persistent differences with healthy controls despite the improvements [57], thus it is likely to think that balance impairments are never completely addressed following THA. These observations are confirmed by the results of the study by Ninomiya et al. [51], which observed a 2.8 times higher fall rate in THA patients compared to healthy controls in the 10 years after surgery. The second aim of this review was to investigate how balance and proprioception are commonly assessed in THA patients. It seems that the most used tasks for balance assessment are those investigating COP displacement or other similar parameters during double- and single-limb stance performed on force platforms or other instrumented devices ( 22 out of the 32 studies in this review). In general, the use of force platforms for the assessment of static and dynamic balance is well accepted in literature given the high reliability of the instrumentation [73]. In the specific case of patients undergoing THA, the use of force platforms together with other devices, such as camera for motion capture or inertial sensors, may provide also additional information on the kinematic of the operated hip joint and the whole lower limb, which may show peculiar abnormalities or compensations negatively affecting for example the non-operated limb or the whole-body posture. Further research is needed to deeply investigate these aspects and how balance changes in the long-term following THA. Regarding proprioception, it was assessed in only two studies $[60,61]$ by means of hip joint repositioning tasks. These tasks are mainly aimed at investigating the joint position sense. It will be useful adding other tasks to investigate proprioception during dynamic tasks. In addition, further research is needed to understand the extent to which abnormal hip proprioception affects whole-body balance above and beyond the other body functions and structures involved in balance ability. It is not possible to draw conclusion on which tests are more indicated in the different times of rehabilitation because of the heterogeneity of the studies. However, some considerations have to be mentioned regarding some of the other tests used for balance assessment. The assessment of dynamic balance by means of asymmetrical loading during squatting and sit-to-standing, as well as loading during walking, needs to consider that balance is not the only variable affecting the results of the measure. Other factors such as muscle strength, post-operative training of loading symmetry, and fear of loading the operated limb might play a role in the performance of those tasks. The same is for tasks such as the functional reach test, used as a measure of balance. Undoubtfully balance is required for the functional reach test, but also muscle strength of hip, back and in general upper body muscles play an important role for a good performance of the test. At the same time, it should be mentioned that muscle strength might contributes per se to balance abilities. In support of this observation, one of the studies included in the present review reported better balance abilities while walking in the early post-surgery in THA patients undergoing a strength training intervention in comparison with patients receiving a usual rehabilitation [71]. In addition, in another study [63] male patients showed better static and dynamic balance abilities than the female counterpart. The author concluded that this difference might be related to between-groups differences in muscle strength.

Regarding surgical approaches used for THA, it is not possible to draw conclusions regarding the best surgical approach for the performance of double- and single-limb stance, because of the contrasting results when THA, HR or large-diameter-THAs patients are compared [ 41-43, 47, 49]. Similarly, it is not possible to draw conclusions for the other functional tasks, such as the timed-up-and-go, the functional reach test or the stepping tasks, because of the paucity of studies [ 41, 62, 64]. Similar findings are observed about hip 
joint proprioception [61], for the differences between patients with monolateral and bilateral THAs [11, 53], and for the differences between patients undergoing THA in the traumatic and elective setting [60]. No differences have been found among patients undergoing anterior or lateral surgical approaches in terms of step test performance [59], of confidence during balance task [59], and of the risk of post-surgical falls [59]. Moreover, no differences were found for balance recovery following sudden perturbations in patients operated on using direct-lateral and antero-lateral surgical approaches, in which the dynamic balancing ability continuously improved in the first 6 months postoperatively [58]. Accordingly, no significant differences between approaches were found for the risk of falls in the first 3 months after surgery [64], as well as for fulfilment of the single-limb balance tasks 2 months following surgery [39]. However, while subjects operated on through the posterior approach showed no significant differences from controls subjects, patients operated on with the anterior or Röttinger approach showed worse balance than controls [39]. In the case of joint capsule preserving posterior approach, the dynamic balancing ability showed a more rapidly improvement across timelines compared to the other two exposures, with no differences in the long-term [59]. However, further evidence is required to confirm these observations.

\section{Balance rehabilitation training}

Although the limited number of studies and the different methodological approach do not allow a univocal conclusion, it could be stated that balance training seems to be effective in all the phases of THA, pre-operative [68], immediate post-surgery $[65,70,72]$ and mediumlog-term $[68,70]$, if specifically structured for balance enhancement and consistent in training volume. In fact, the two studies reporting no higher benefits in the intervention group, assessed balance following a nonbalance specific training [66], or after a minimal intervention strategy, demanding minimal training effort exercises [69]. Therefore, balance training interventions for patients with THA should be well structured in terms of type and volume. Just one study suggest that an early strength training intervention leads to better balance while walking in the early post-surgery [71], thus it seems beneficial for the prevention of early re-injuries. No conclusion can be drawn regarding the best training options for the different post-surgical phases.

\section{Limitations}

The main limitation of this literature revision was that it was difficult to sum up a conclusion for a number of the investigated points since a limited number of studies were available and most of them were excluded during the selection process mainly because of low quality or because data of THA patients were mixed to data of patients with other orthopaedic impairments. Further, in a number of studies there was not a control group of healthy matched participants to make comparisons with normative data. It is paramount for future studies to eliminate sources of bias and improve studies quality. In addition, since it has been reported that balance abnormalities lead to an increase in the risk of falls [13], another point which should be investigated in future studies, is the relationship between the introduction of specific balance training interventions in the rehabilitation following THA, and the long-term effects on the risk of falls. The studies included in the present review did not report this information, thus results should be considered in light of this limitation. Finally, for some of the points discussed in this review few studies exist. Thus, caution is needed before the modification of clinical practice, and it seems thus essential to conduct further high-quality research to increase the amount of evidences.

\section{Conclusion}

Even if a firm conclusion cannot be drawn because of the heterogeneity of the studies and the reduced number of evidences, an improvement in balance abilities is observed following THA surgery in comparison with the pre-operative performance, and balance abnormalities persists for year after surgery, with THA patients showing an increased risk for falls. Since it seems that balance training is effective in all the rehabilitation phases if specifically structured for balance enhancement and consistent in training volume, it is not clear if long-term balance impairments are related to the lack of appropriate training interventions, or to the sensory-motor impairments related to THA surgery. It remains unclear which assessments are more appropriate for the different rehabilitation phases, and if differences exist between the different surgical procedure used for THA. Moreover, only two studies exist assessing hip joint proprioception. Further research is needed to better investigate these gaps in balance and proprioception assessment and training in THA patients.

\footnotetext{
Abbreviations

THA: Total Hip Arthroplasty; DAA: Direct Anterior Approach; PRISMA: Preferred Reporting Items for Systematic Reviews and Meta-Analyses; COP: Center Of Pressure; HR: Hip Resurfacing; ML: Medial-Lateral; AP: AnteriorPosterior; COM: Center Of Mass; FP: Frontal Plane; ABC: Activities-specific Balance Confidence Scale; FROP-Com: Falls Risk for Older People in a Community setting.
} 


\section{Supplementary Information}

The online version contains supplementary material available at https://doi. org/10.1186/s12891-021-04919-w.

\section{Additional file 1: Appendix 1. \\ Additional file 2: Appendix 2.}

Additional file 3: Appendix 3.

\section{Acknowledgments}

Not applicable.

\section{Authors' contributions}

MGB, ADM and LL conceived and planned the study, and all others approved the study protocol. FC, FB and NS performed the searches and the selection of papers. FC, FB, NS and LL did the data extraction and risk of bias analysis. $\mathrm{LL}$ wrote the draft of the manuscript which all authors critically revised. All authors have read and approved the final version of the manuscript.

\section{Funding}

No funding has been received for this study.

\section{Availability of data and materials}

All the materials related the current study are available from the corresponding author upon reasonable request.

\section{Declarations}

\section{Ethics approval and consent to participate}

Not applicable.

\section{Consent for publication}

Not applicable.

\section{Competing interests}

The authors declare no competing interests.

\section{Author details}

${ }^{1}$ Physical Medicine and Rehabilitation Unit, IRCCS - Istituto Ortopedico Rizzoli, Via Giulio Cesare Pupilli 1, 40136 Bologna, Italy. ${ }^{2}$ Department of Biomedical and Neuromotor Sciences, University of Bologna, Bologna, Italy. ${ }^{3}$ Orthopaedic Clinic, IRCSS- Istituto Ortopedico Rizzoli, Bologna, Italy.

Received: 30 April 2021 Accepted: 28 November 2021 Published online: 20 December 2021

\section{References}

1. Shan L, Shan B, Graham D, et al. Total hip replacement: a systematic review and meta-analysis on mid-term quality of life. Osteoart Cart. 2014;22:389-406. https://doi.org/10.1016/j.joca.2013.12.006.

2. Bahl JS, Nelson MJ, Taylor M, et al. Biomechanical changes and recovery of gait function after total hip arthroplasty for osteoarthritis: a systematic review and meta-analysis. Osteoart Cart. 2018;26(7):847-63. https://doi. org/10.1016/j.joca.2018.02.897.

3. Nguyen UDT, Perneger T, Franklin PD, et al. Improvement in mental health following total hip arthroplasty: the role of pain and function. BMC Musculoskelet Disord. 2019;20:307. https://doi.org/10.1186/ s12891-019-2669-y.

4. Proske U, Gandevia SC. The proprioceptive senses: their roles in signaling body shape, body position and movement, and muscle force. Physiol Rev. 2012;92(4):1651-97. https://doi.org/10.1152/physrev.00048. 2011

5. Miura N, Tagomori K, Ikutomo H, et al. Asymmetrical loading during sit-tostand movement in patients 1 year after total hip arthroplasty. Clin Biomech. 2018;57:89-92. https://doi.org/10.1016/j.clinbiomech.2018.06.017.
6. Bragonzoni L, Rovini E, Barone G, et al. How proprioception changes before and after total knee arthroplasty: a systematic review. Gait Posture. 2019;72:1-11. https://doi.org/10.1016/j.gaitpost.2019.05.005.

7. Hillsdon M, Foster C. What are the health benefits of muscle and bone strengthening and balance activities across life stages and specific health outcomes? J Frail Sarcop Fall. 2018;3(2):66 https://doi.org/10.22540/ JFSF-03-066.

8. Bobić Lucić L, Grazio S. Impact of balance confidence on daily living activities of older people with knee osteoarthritis with regard to balance, physical function, pain, and quality of life-a preliminary report. Clin Gerontol. 2018;41(4):357-65. https://doi.org/10.1080/07317115. 2018.1453907.

9. Dunsky A. The effect of balance and coordination exercises on quality of life in older adults: a mini-review. Front Aging Neurosc. 2019;11:318. https://doi.org/10.3389/fnagi.2019.00318.

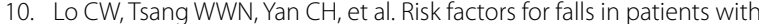
total hip arthroplasty and total knee arthroplasty: a systematic review and meta-analysis. Osteoart Cart. 2019;27(7):979-93. https://doi.org/10. 1016/j.joca.2019.04.006

11. Temporiti F, Zanotti G, Furone R, et al. Functional and postural recovery after bilateral or unilateral total hip arthroplasty. J Electromyog Kinesiol. 2019:48:205-11. https://doi.org/10.1016/j.jelekin.2019.08.002.

12. De Lima F, Fernandes DA, Melo G, et al. Effects of total hip arthroplasty for primary hip osteoarthritis on postural balance: a systematic review. Gait Posture. 2019;73:52-64. https://doi.org/10.1016/j.gaitpost.2019.07.124.

13. Hunter SW, Bobos P, Somerville L, et al. Prevalence and risk factors of falls in adults 1 year after total hip arthroplasty for osteoarthritis. Am J Phys Med Rehab. 2020;99:853-7. https://doi.org/10.1097/PHM.0000000000 001456.

14. Ikutomo H, Nagai K, Tagomori K, et al. Incidence and circumstances of falls in women before and after total hip arthroplasty: a prospective cohort study. J Arthropl. 2018;33(7):2268-72. https://doi.org/10.1016/j. arth.2018.02.006.

15. Petis $\mathrm{S}$, Howard $J \mathrm{~L}$, Lanting BL, et al. Surgical approach in primary total hip arthroplasty: anatomy, technique and clinical outcomes. Can J Surg. 2015;58(2):128. https://doi.org/10.1503/cjs.007214.

16. Den Daas A, Reitsma EA, Knobben BAS, et al. Patient satisfaction in different approaches for total hip arthroplasty. Orthop Traumatol Surg Res. 2019;105(7):1277-82. https://doi.org/10.1016/j.otsr.2019.08.003.

17. Moyer R, Lanting B, Marsh J, et al. Postoperative gait mechanics after total hip arthroplasty: a systematic review and meta-analysis. JBJS Rev. 2018:6(11):e1. https://doi.org/10.2106/JBJS.RVW.17.00133.

18. Putananon C, Tuchinda H, Arirachakaran A, et al. Comparison of direct anterior, lateral, posterior and posterior-2 approaches in total hip arthroplasty: network meta-analysis. Eur J Orthop Surg Traumatol. 2018;28:25567. https://doi.org/10.1007/s00590-017-2046-1.

19. Migliorini F, Trivellas A, Eschweiler J, et al. Hospitalization length, surgical duration, and blood lost among the approaches for total hip arthroplasty: a Bayesian network meta-analysis. Musculoskelet Surg. 2020;104:257-66. https://doi.org/10.1007/s12306-020-00657-9.

20. Domínguez-Navarro F, Igual-Camacho C, Silvestre-Muñoz A, et al. Effects of balance and proprioceptive training on total hip and knee replacement rehabilitation: a systematic review and meta-analysis. Gait Posture 2018;62:68-74. https://doi.org/10.1016/j.gaitpost.2018.03.003.

21. Cronin P, Ryan F, Coughlan M. Undertaking a literature review: a step-bystep approach. Br J Nurs. 2008;17(1):38-43. https://doi.org/10.12968/bjon. 2008.17.1.28059.

22. Gregory AT, Denniss AR. An introduction to writing narrative and systematic reviews - tasks, tips and traps for aspiring authors. Heart, Lung and Circulation. 2018:27(7):893-8. https://doi.org/10.1016/j.hlc.2018.03.027.

23. Learmonth ID, Young C, Rorabeck C. The operation of the century: total hip replacement. Lancet. 2007:370(9597):1508-19. https://doi.org/10. 1016/S0140-6736(07)60457-7

24. Downs SH, Black N. The feasibility of creating a checklist for the assessment of the methodological quality both of randomised and nonrandomised studies of health care interventions. J Epidemiol Community Health. 1998;52(6):377-84. https://doi.org/10.1136/jech.52.6.377.

25. Munn J, Sullivan SJ, Schneiders AG. Evidence of sensorimotor deficits in functional ankle instability: a systematic review with meta-analysis. J Sci Med Sport. 2010;13:2-12. https://doi.org/10.1016/j.jsams.2009.03.004 
26. Landis JR, Koch GG. The measurement of observer agreement for categorical data. Biometrics. 1977;33:159-74. https://doi.org/10.2307/ 2529310.

27. Jogi P, Zecevic A, Overend TJ, et al. Force-plate analyses of balance following a balance exercise program during acute post-operative phase in individuals with total hip and knee arthroplasty: a randomized clinical trial. SAGE Open Med. 2016;4:2050312116675097. https://doi.org/10. $1177 / 2050312116675097$.

28. Boeer J, Mueller O, Krauss I, et al. Effects of a sensory-motor exercise program for older adults with osteoarthritis or prosthesis of the hip using measurements made by the Posturomed oscillatory platform. J Ger Phys Ther. 2010;33(1):10-5.

29. Jogi P, Overend TJ, Spaulding SJ, et al. Effectiveness of balance exercises in the acute post-operative phase following total hip and knee arthroplasty: a randomized clinical trial. SAGE Open Med. 2015;3:2050312115570769. https://doi.org/10.1177/2050312115570769.

30. Batting M, Barker KL. Reliability and validity of the Four-Square step test in patients with hip osteoarthritis before and after total hip replacement. Physiother. 2019;105(2):244-53. https://doi.org/10.1016/j.physio.2018.07. 014.

31. Pohl T, Brauner T, Wearing $S$, et al. Effects of sensorimotor training volume on recovery of sensorimotor function in patients following lower limb arthroplasty. BMC Musculoskel Disord. 2015;16(1):1-9. https://doi.org/10. 1186/s12891-015-0644-9.

32. Jogi P, Spaulding SJ, Zecevic AA, et al. Comparison of the original and reduced versions of the Berg balance scale and the Western Ontario and McMaster universities osteoarthritis index in patients following hip or knee arthroplasty. Physiother Canada. 2011;63(1):107-14. https://doi.org/ 10.3138/ptc.2009-26.

33. Slaven EJ. Prediction of functional outcome at six months following total hip arthroplasty. Phys Ther. 2012;92(11):1386-94. https://doi.org/10.2522/ ptj.20110484

34. Calo L, Rabini A, Picciotti PM, et al. Postural control in patients with total hip replacement. Eu J Phys Rehab Med. 2009;45(3):327-34.

35. Esposito F, Freddolini M, Latella $L$, et al. The influence of the crutch setup on stability and weight-bearing parameters in post total hip replacement surgery patients during quiet standing. Disabil Rehab Assist Tech. 2018;13(4):373-8. https://doi.org/10.1080/17483107.2017.1328617.

36. Majewski M, Bischoff-Ferrari HA, Grüneberg C, et al. Improvements in balance after total hip replacement. J Bone Joint Surg Brit. 2005;87(10):133743. https://doi.org/10.1302/0301-620X.87B10.16605.

37. Merle J, Rougier P, Belaid D, et al. Is early weight bearing resumption beneficial after total hip replacement? Orthop Traumatol Surg Res. 2009;95(2):127-33. https://doi.org/10.1016/j.otsr.2008.12.001.

38. Rougier P, Belaid D, Cantalloube S, et al. Quiet postural control of patients with total hip arthroplasty following joint arthritis. Mot Control. 2008;12(2):136-50. https://doi.org/10.1123/mcj.12.2.136.

39. Van Driessche S, Billuart F, Martinez $L$, et al. Short-term comparison of postural effects of three minimally invasive hip approaches in primary total hip arthroplasty: direct anterior, posterolateral and Röttinger. Orthop Traumatol Surg Res. 2016;102(6):729-34. https://doi.org/10.1016/j.otsr. 2016.05.003.

40. Chang CJ, Lin NL, Lee MS, et al. Recovery of posture stability at different foot placements in patients who underwent minimally invasive total hip arthroplasty: a one-year follow-up study. Biomed Res Int. 2015;463792. https://doi.org/10.1155/2015/463792.

41. Lavigne M, Therrien M, Nantel J, et al. The John Charnley award: the functional outcome of hip resurfacing and large-head THA is the same: a randomized, double-blind study. Clin Orthop Rel Res. 2010;468(2):326-36. https://doi.org/10.1007/s11999-009-0938-z.

42. Nantel J, Termoz N, Centomo H, et al. Postural balance during quiet standing in patients with total hip arthroplasty and surface replacement arthroplasty. Clin Biomech. 2008;23(4):402-7. https://doi.org/10.1016/j. clinbiomech.2007.10.011.

43. Nantel J, Termoz N, Ganapathi M, et al. Postural balance during quiet standing in patients with total hip arthroplasty with large diameter femoral head and surface replacement arthroplasty. Arch Phys Med Rehab. 2009;90(9):1607-12. https://doi.org/10.1016/j.apmr.2009.01.033.

44. Pop T, Szymczyk D, Majewska J, et al. The assessment of static balance in patients after total hip replacement in the period of 2-3 years after surgery. Biomed Res Int. 2018. https://doi.org/10.1155/2018/3707254.
45. Quagliarella L, Sasanelli N, Monaco V, et al. Relevance of orthostatic posturography for clinical evaluation of hip and knee joint arthroplasty patients. Gait Posture. 2011;34(1):49-54. https://doi.org/10.1016/j.gaitp ost.2011.03.010.

46. Rasch A, Dalén N, Berg HE. Muscle strength, gait, and balance in 20 patients with hip osteoarthritis followed for 2 years after THA. Acta Orthop. 2010;81(2):183-8. https://doi.org/10.3109/17453671003793204.

47. Szymanski C, Thouvarecq R, Dujardin F, et al. Functional performance after hip resurfacing or total hip replacement: a comparative assessment with non-operated subjects. Orthop Traumatol Surg Res. 2012;98(1):1-7. https://doi.org/10.1016/j.otsr.2011.10.006.

48. Eyvazov K, Eyvazov B, Basar S, et al. Effects of total hip arthroplasty on spinal sagittal alignment and static balance: a prospective study on 28 patients. Eu Spine J. 2016;25(11):3615-21. https://doi.org/10.1007/ s00586-016-4696-9.

49. Larkin B, Nyazee H, Motley J, et al. Hip resurfacing does not improve proprioception compared with THA. Clin Orthop Rel Res. 2014;472(2):555-61. https://doi.org/10.1007/s11999-013-3082-8.

50. Butler RJ, Thiele RAR, Barnes $C L$, et al. Unipedal balance is affected by lower extremity joint arthroplasty procedure 1 year following surgery. J Arthrop. 2015;30(2):286-9. https://doi.org/10.1016/j.arth.2014.08.031.

51. Ninomiya K, Hirakawa K, Ikeda T, et al. Patients 10 years after total hip arthroplasty have the deficits in functional performance, physical activity, and high fall rate compared to healthy adults. Phys Ther Res. 2018;21(2):53-8. https://doi.org/10.1298/ptr.E9941.

52. Wareńczak A, Lisiński P. Does total hip replacement impact on postural stability? BMC Musculoskel Disord. 2019;20(1):1-9. https://doi.org/10. 1186/s12891-019-2598-9.

53. Talis VL, Grishin AA, Solopova IA, et al. Asymmetric leg loading during sitto-stand, walking and quiet standing in patients after unilateral total hip replacement surgery. Clin Biomech. 2008;23(4):424-33. https://doi.org/ 10.1016/j.clinbiomech.2007.11.010.

54. Esbjörnsson AC, Naili JE. Functional movement compensations persist in individuals with hip osteoarthritis performing the five times sit-to-stand test 1 year after total hip arthroplasty. J Orthop Surg Res. 2020;15:1-8. https://doi.org/10.1186/s13018-020-01663-0.

55. Brauner T, Wearing S, Rämisch E, et al. Can measures of limb loading and dynamic stability during the squat maneuver provide an index of early functional recovery after unilateral total hip arthroplasty? Arc Phys Med Rehab. 2014;95(10):1946-53. https://doi.org/10.1016/j.apmr.2014.06.003.

56. Sliwinski MM, Sisto SA, Batavia M, et al. Dynamic stability during walking following unilateral total hip arthroplasty. Gait Posture. 2004;19(2):141-7. https://doi.org/10.1016/S0966-6362(03)00039-0.

57. Lugade $V$, Klausmeier $V$, Jewett $B$, et al. Short-term recovery of balance control after total hip arthroplasty. Clin Orthop Rel Res. 2008;466(12):3051-8. https://doi.org/10.1007/s11999-008-0488-9.

58. Holnapy G, Kiss RM. Impact of the method of exposure in total hip arthroplasty on balancing ability in response to sudden unidirectional perturbation in the first six months of the postoperative period. J Electromyog Kinesiol. 2013;23(3):727-33. https://doi.org/10.1016/j.jelekin. 2013.04.005.

59. Hunter SW, Bobos P, Somerville L, et al. Comparison of functional and patient-reported outcomes between direct anterior and lateral surgical approach one-year after total hip arthroplasty in a Canadian population: a cross-sectional study. J Orthop. 2020;19:36-40. https://doi.org/10. 1016/j.jor.2019.11.004.

60. Jo S, Park SB, Kim MJ, et al. Comparison of balance, proprioception and skeletal muscle mass in total hip replacement patients with and without fracture: a pilot study. Ann Rehab Med. 2016;40(6):1064. https://doi.org/ 10.5535/arm.2016.40.6.1064.

61. Wang C, Zhu LB, Lu HM, et al. Clinical evaluation of hip joint position sense in patients with hip resurfacing arthroplasty versus conventional total hip arthroplasty. Int J Clin Exp Med. 2016;9(10):19809-14.

62. Wareńczak A, Lisiński P. Body balance a few years after total hip replacement. Acta Bioeng Biomec. 2020;22(1):87-96.

63. Queen RM, Schmitt D. Sex-specific difference in dynamic balance following Total hip replacement. Innov Aging. 2021;5(2):igab019. https://doi. org/10.1093/geroni/igab019.

64. Ulivi M, Orlandini L, Vitale JA, et al. Direct superior approach versus posterolateral approach in total hip arthroplasty: a randomized controlled trial on early outcomes on gait, risk of fall, clinical and self-reported 
measurements. Acta Orthop. 2021;92(3):274-9. https://doi.org/10.1080/ 17453674.2020 .1865633$.

65. Aprile I, lacovelli C, Cruciani A, et al. Technological rehabilitation versus conventional rehabilitation following hip replacement: a prospective controlled study. J Back Musculoskel Rehab. 2020;33(4):561-8. https://doi. org/10.3233/BMR-181211.

66. Nelson M, Bourke M, Crossley K, et al. Telerehabilitation is non-inferior to usual care following total hip replacement-a randomized controlled non-inferiority trial. Physiother. 2020;107:19-27. https://doi.org/10.1016/j. physio.2019.06.006.

67. Trudelle-Jackson E, Smith SS. Effects of a late-phase exercise program after total hip arthroplasty: a randomized controlled trial. Arc Phys Med Rehab. 2004;85(7):1056-62. https://doi.org/10.1016/j.apmr.2003.11.022.

68. Zeng R, Lin J, Wu S, et al. A randomized controlled trial: preoperative home-based combined tai chi and strength training (TCST) to improve balance and aerobic capacity in patients with total hip arthroplasty (THA). Arch Gerontol Ger. 2015;60(2):265-71. https://doi.org/10.1016/j. archger.2014.11.009.

69. Bitterli R, Sieben JM, Hartmann M, et al. Pre-surgical sensorimotor training for patients undergoing total hip replacement: a randomised controlled trial. Int J Sports Med. 2011;32(09):725-32. https://doi.org/10.1055/s0031-1271696.

70. Pethe-Kania K, Opara JA, Kania D, et al. The follow-up posturography in rehabilitation after total hip arthroplasty. Acta Bioeng Biomec. 2017;19(1):97-104.

71. Winther SB, Foss OA, Klaksvik J, Husby VS. Increased muscle strength limits postural sway during daily living activities in total hip arthroplasty patients. Am J Phys Med Rehab. 2020;99(7):608-12. https://doi.org/10. 1097/PHM.0000000000001382.

72. Shabana M, Rahim AAAE, Abdelhakiem NM, et al. Impact of dynamic balance training on hip musculature moments and stability index in programming total hip arthroplasty. Eur J Mol Clin Med. 2021;8(2):839-49.

73. Li Z, Liang YY, Wang $L$, et al. Reliability and validity of center of pressure measures for balance assessment in older adults. J Phys Ther Sci. 2016;28(4):1364-7.

\section{Publisher's Note}

Springer Nature remains neutral with regard to jurisdictional claims in published maps and institutional affiliations.

Ready to submit your research? Choose BMC and benefit from:

- fast, convenient online submission

- thorough peer review by experienced researchers in your field

- rapid publication on acceptance

- support for research data, including large and complex data types

- gold Open Access which fosters wider collaboration and increased citations

- maximum visibility for your research: over $100 \mathrm{M}$ website views per year

At $\mathrm{BMC}$, research is always in progress.

Learn more biomedcentral.com/submissions 